\title{
Two-season Atacama Cosmology Telescope polarimeter lensing power spectrum
}

Blake D. Sherwin, ${ }^{1}$ Alexander van Engelen, ${ }^{2}$ Neelima Sehgal,${ }^{3}$ Mathew Madhavacheril,,${ }^{4,3}$ Graeme E. Addison, ${ }^{5}$ Simone Aiola,${ }^{6,7,8}$ Rupert Allison, ${ }^{9}$ Nicholas Battaglia ${ }^{4}$ Daniel T. Becker, ${ }^{10}$ James A. Beall, ${ }^{10}$ J. Richard Bond, ${ }^{2}$ Erminia Calabrese, ${ }^{11}$ Rahul Datta, ${ }^{12}$ Mark J. Devlin, ${ }^{13}$ Rolando Dünner, ${ }^{14}$ Joanna Dunkley, ${ }^{6,411}$ Anna E. Fox, ${ }^{10}$ Patricio Gallardo, ${ }^{15}$ Mark Halpern, ${ }^{16}$ Matthew Hasselfield, ${ }^{17,18}$ Shawn Henderson, ${ }^{15}$ J. Colin Hill, ${ }^{19}$ Gene C. Hilton, ${ }^{10}$ Johannes Hubmayr, ${ }^{10}$ John P. Hughes, ${ }^{20}$ Adam D. Hincks, ${ }^{21}$ Renée Hlozek, ${ }^{22}$ Kevin M. Huffenberger, ${ }^{23}$ Brian Koopman, ${ }^{15}$ Arthur Kosowsky, ${ }^{7,8}$ Thibaut Louis, ${ }^{24}$ Loïc Maurin, ${ }^{14}$ Jeff McMahon, ${ }^{12}$ Kavilan Moodley, ${ }^{25}$ Sigurd Naess, ${ }^{26,11}$

Federico Nati, ${ }^{13}$ Laura Newburgh, ${ }^{22}$ Michael D. Niemack, ${ }^{15}$ Lyman A. Page, ${ }^{6}$ Jonathan Sievers, ${ }^{27}$ David N. Spergel, ${ }^{4,26}$ Suzanne T. Staggs, ${ }^{6}$ Robert J. Thornton, ${ }^{28,13}$ Jeff Van Lanen, ${ }^{10}$ Eve Vavagiakis, ${ }^{15}$ and Edward J. Wollack ${ }^{29}$

${ }^{1}$ Berkeley Center for Cosmological Physics, Lawrence Berkeley National Laboratory, Berkeley, California 94720, USA

${ }^{2}$ Canadian Institute for Theoretical Astrophysics, University of Toronto, Toronto, Ontario, Canada M5S $3 \mathrm{H} 8$

${ }^{3}$ Physics and Astronomy Department, Stony Brook University, Stony Brook, New York 11794, USA

${ }^{4}$ Department of Astrophysical Sciences, Peyton Hall, Princeton University, Princeton, New Jersey 08544, USA

${ }^{5}$ Dept. of Physics and Astronomy, The Johns Hopkins University, 3400 North Charles Street, Baltimore, Maryland 21218-2686, USA

${ }^{6}$ Joseph Henry Laboratories of Physics, Jadwin Hall, Princeton University, Princeton, New Jersey 08544, USA

${ }^{7}$ Department of Physics and Astronomy, University of Pittsburgh, Pittsburgh, Pennsylvania 15260, USA

${ }^{8}$ Pittsburgh Particle Physics, Astrophysics, and Cosmology Center, University of Pittsburgh, Pittsburgh, Pennsylvania 15260, USA

${ }^{9}$ Institute of Astronomy, Cambridge, Madingley Road, Cambridge CB3 OHA, United Kingdom ${ }^{10}$ NIST Quantum Devices Group, 325 Broadway Mailcode 817.03, Boulder, Colorado 80305, USA

${ }^{11}$ Sub-Department of Astrophysics, University of Oxford, Keble Road, Oxford OX1 3RH, United Kingdom

${ }^{12}$ Department of Physics, University of Michigan, Ann Arbor, Michigan 48103, USA

${ }^{13}$ Department of Physics and Astronomy, University of Pennsylvania, Philadelphia, Pennsylvania 19104, USA

${ }^{14}$ Instituto de Astrofísica and Centro de Astro-Ingeniería, Facultad de Física,

Pontificia Universidad Católica de Chile, Av. Vicuña Mackenna 4860, 7820436 Macul, Santiago, Chile

${ }^{15}$ Department of Physics, Cornell University, Ithaca, New York 14853, USA

${ }^{16}$ Department of Physics and Astronomy, University of British Columbia, Vancouver, British Columbia, Canada V6T $1 \mathrm{Z4}$

${ }^{17}$ Department of Astronomy and Astrophysics, The Pennsylvania State University, University Park, Pennsylvania 16802, USA

${ }^{18}$ Institute for Gravitation and the Cosmos, The Pennsylvania State University, University Park, Pennsylvania 16802

${ }^{19}$ Department of Astronomy, Pupin Hall, Columbia University, New York, NY 10027, USA

${ }^{20}$ Department of Physics and Astronomy, Rutgers, The State University of New Jersey, Piscataway, New Jersey 08854-8019, USA

${ }^{21}$ Department of Physics, University of Rome "La Sapienza", Piazzale Aldo Moro 5, I-00185 Rome, Italy

${ }^{22}$ Dunlap Institute, University of Toronto, 50 St. George Street, Toronto, Ontario, Canada M5S 3H4, USA

${ }^{23}$ Department of Physics, Florida State University, Tallahassee Florida 32306, USA

${ }^{24}$ UPMC Univ Paris 06, UMR7095, Institut d'Astrophysique de Paris, F-75014 Paris, France

${ }^{25}$ Astrophysics and Cosmology Research Unit, School of Mathematics, Statistics and Computer Science, University of KwaZulu-Natal, Durban 4041, South Africa

${ }^{26}$ Center for Computational Astrophysics, 162 5th Avenue, New York, New York 10003, USA

${ }^{27}$ Astrophysics and Cosmology Research Unit, School of Chemistry and Physics,

University of KwaZulu-Natal, Durban, South Africa National Institute for Theoretical Physics, Durban 4000, KwaZulu-Natal, South Africa

${ }^{28}$ Department of Physics, West Chester University of Pennsylvania, West Chester, Pennsylvania 19383, USA

${ }^{29}$ NASA/Goddard Space Flight Center, Greenbelt, Maryland 20771, USA

(Received 10 December 2016; published 21 June 2017) 
We report a measurement of the power spectrum of cosmic microwave background (CMB) lensing from two seasons of Atacama Cosmology Telescope polarimeter (ACTPol) CMB data. The CMB lensing power spectrum is extracted from both temperature and polarization data using quadratic estimators. We obtain results that are consistent with the expectation from the best-fit Planck $\Lambda$ CDM model over a range of multipoles $L=80-2100$, with an amplitude of lensing $A_{\text {lens }}=1.06 \pm 0.15 \quad$ (stat) $\pm 0.06 \quad$ (sys) relative to Planck. Our measurement of the CMB lensing power spectrum gives $\sigma_{8} \Omega_{m}^{0.25}=0.643 \pm 0.054$; including baryon acoustic oscillation scale data, we constrain the amplitude of density fluctuations to be $\sigma_{8}=0.831 \pm 0.053$. We also update constraints on the neutrino mass sum. We verify our lensing measurement with a number of null tests and systematic checks, finding no evidence of significant systematic errors. This measurement relies on a small fraction of the ACTPol data already taken; more precise lensing results can therefore be expected from the full ACTPol data set.

DOI: 10.1103/PhysRevD.95.123529

\section{INTRODUCTION}

The large-scale structure of the Universe contains a wealth of information about the early Universe, neutrinos, dark energy, and other physics that we are only beginning to extract. While measurements of large-scale structure using galaxies, quasars, Lyman- $\alpha$ absorbers, and other tracers continue to give great insight, these measurements are somewhat complicated by their reliance on biased probes of the mass distribution. In contrast, gravitational lensing directly probes all mass, including dark matter.

The cosmic microwave background $(\mathrm{CMB})$ radiation has unique advantages as a background light source for the study of gravitational lensing. CMB photons originate from the last scattering surface at $z \simeq 1100$ and experience gravitational lensing deflections from large-scale structure along their paths to our telescopes. Hence, CMB lensing encodes information about nearly all the mass fluctuations in the Universe, with most of the signal arising between $z=0.5$ and $z=3[1-4]$. The fact that much of the lensing signal originates from high redshifts and large scales means that the signal is simple to model, with most complications from nonlinear evolution and baryonic physics negligible at current and near-future precision [5]. An additional simplifying feature is that the primordial CMB source is well understood, with a known redshift origin and simple statistical properties. Measurements of the CMB lensing signal therefore can serve as accurate probes of cosmology.

Given current measurement precision, the CMB lensing field can be modeled as Gaussian, so the power spectrum describes all its cosmological information; for future surveys, higher-order statistics may add information [6-8]. As the CMB lensing power spectrum probes the projected mass distribution, it is sensitive to both the growth of structure and the geometry of the Universe. Hence it is capable of constraining parameters such as neutrino mass, the amplitude of density fluctuations, curvature, and dark energy.

Measurements of the lensing power spectrum have only recently become possible with the advent of highresolution, low-noise $\mathrm{CMB}$ telescopes such as the Atacama
Cosmology Telescope (ACT) [9], the South Pole Telescope (SPT) [10], and the Planck satellite [11]. Following earlier cross-correlation results from WMAP [12,13], the ACT team made the first measurement of the lensing power spectrum [14] and was able to confirm the existence of dark energy based on only CMB observations [15]. The SPT Collaboration was able to make a more sensitive measurement of temperature lensing [16]. The POLARBEAR Collaboration made the first measurements of the lensing power spectrum using polarization data [17,18], following the first detection of polarization lensing in crosscorrelation using SPTpol and Herschel [19]. Subsequently, the SPTpol [20] and BICEP2/Keck [21] teams presented measurements of polarization and temperature lensing power spectra with increased precision. The Planck team has made the current highest precision measurement of the lensing power spectrum: a $40 \sigma$ detection significance in their latest release $[22,23]$.

While the Planck lensing power spectrum is generally in agreement with $\Lambda \mathrm{CDM}$, the authors report some tension at small scales, with a null test failure at the $\sim 2.9 \sigma$ level [23]. In addition, several recent measurements using galaxy lensing and galaxy clusters have reported an amplitude of density fluctuations lower than that found with Planck lensing data, or Planck primary CMB data, at $2 \sigma$ or higher significance, e.g. [24]. The main goals of this work are to present a new measurement of the lensing power spectrum, to independently constrain parameters such as the neutrino mass, and to introduce the ACTPol lensing pipeline. The possibility of testing both the Planck lensing results and any potential tensions between different measurements of the amplitude of structure provides additional motivation for our work.

This paper presents new measurements of the CMB lensing power spectrum using the first two seasons of ACTPol nighttime data and the resulting constraints on cosmological parameters. The current measurement relies on only $12 \%$ of the usable ACTPol data already taken [25]. Future measurements using the full ACTPol data set will thus have higher precision, and our paper serves also as an exposition of the pipeline that we will use for this future 
work. Our analysis follows first-season ACTPol lensing results, which include a cross-correlation with maps of the cosmic infrared background fluctuations [26]; a crosscorrelation with radio sources to constrain their bias [27]; and a detection of lensing by dark matter halos by stacking on spectroscopic galaxies [28]. In Sec. II, we describe the data and simulations we use in our analysis. In Sec. III, we describe our pipeline for measuring the CMB lensing power. We present our results in Sec. IV and verify our measurements with systematic estimates and null tests described in Sec. V. We discuss the implications of our results for cosmological parameters in Sec. VI and conclude in Sec. VII.

\section{DATA AND SIMULATIONS}

$\mathrm{ACT}$ is a 6-meter diameter $\mathrm{CMB}$ telescope operating in the Atacama Desert in Chile. The ACTPol receiver fitted to this telescope consists of three arrays of superconducting transition edge sensor bolometers, sensitive to both temperature and polarization; see [29] for details on the instrument. ACTPol observed the sky at a frequency of $149 \mathrm{GHz}$ in the first two years of the survey. The observations, data reduction and mapmaking are as described in the most recent ACTPol power spectrum analysis of Louis et al. [30], hereafter L16 (see also the previous analysis [31]).

We use data taken in seasons 1 and 2 from three regions: D5 (57 $\mathrm{deg}^{2}$ at an effective white noise level of $12 \mu \mathrm{K}$-arcmin) and D6 (71 deg at $10.5 \mu \mathrm{K}$-arcmin), both of which are contained within a larger region, D56 $\left(626 \mathrm{deg}^{2}\right.$ at $17 \mu \mathrm{K}$-arcmin) ${ }^{1}$; see [30] for full noise spectra. These three regions are analyzed separately, because the significant variation in map depth would otherwise cause large statistical anisotropy that could be challenging to simulate and subtract accurately. Because the deep survey regions are located entirely within the wide survey footprint, the three different maps cannot be treated as statistically independent in our analysis.

Each of the fields is further processed to reduce the effect of resolved point sources and bright Sunyaev-Zel'dovich (SZ) clusters. Our method for this follows the first-season analysis described in van Engelen et al. [26]. First, we template subtract the detected point sources to a flux limit of $5 \mathrm{mJy}$. In the temperature maps, we additionally in-paint extended galaxy cluster candidates detected at greater than $5 \sigma$ significance (numbering 98 in D56), together with a small number (14 from two map-based catalogs from D56) of irregular, residual point sources detected at greater than $5 \sigma$ significance. In the polarization maps, we mask sources detected at 20 $\sigma$ (290 from two combined D56 catalogs). In both cases we perform the in-painting using constrained Gaussian realizations of $\mathrm{CMB}$ and noise [32]; the mask

\footnotetext{
${ }^{1}$ Although the maps are identical to those analyzed in [30], the boundaries of the analyzed regions differ slightly, leading to slightly different areas for each patch.
}

radii for this in-paint procedure are 5 ' for the clusters and the polarized sources, and 15' for the irregular sources. We apodize our maps using a mask constructed from a product of the weight map, smoothed with a Gaussian of width $l=1200$ in Fourier space, and a cosine-squared edge rolloff of total width $1.7^{\circ}$, where the weights are proportional to the number of detector hits on each map pixel. All maps are deconvolved by the appropriate beams. The resulting polarization maps in Stokes parameters Q and $U$ are then transformed to the $E-B$ basis using the pure- $E B$ method [33]. This method has been found to perform well for lensing reconstruction in [34].

Our simulations are generated as described in $[14,26]$. To construct the signal component of our simulations, we create appropriately correlated, Gaussian-distributed $T, Q$, and $U$ primordial CMB maps using the best-fit parameters of [35]. We then lens the maps with a Gaussian lensing potential using the algorithm described in [36]. We add Gaussian foreground power matching that from ACT observations as described in [26]. After convolving with the appropriate beam, each field is cut out of the larger CMB map; this ensures that the cut-out fields are correlated in the same way as our observed sky areas.

We construct the noise component of our simulations using the map hit-count and noise statistics from the data set as follows. We make the map noise approximately isotropic by multiplying each pixel by the square root of the number of observations in that pixel; we then use four independent splits of the data to obtain a two-dimensional power spectral density, measuring it by subtracting the mean intersplit cross-spectrum from the mean autospectrum. This power spectral density is then used to seed Gaussian random noise maps with the correct two-dimensional power spectral density. The spatial inhomogeneity of noise levels over the map is modeled by dividing the simulations by the square root of the number of observations in each pixel.

After adding the noise and signal components together, the simulations are apodized and transformed into the $E$ and $B$ polarization basis in exactly the same manner as the data. We generate 400 simulations of each field using this method. The full simulation power spectra were found to match those of the data to within $\approx 5 \%$ (the high-statisticalweight temperature map of D56 having the best match of $3 \%$, and the low-weight D5/D6 polarization maps having the worst match to within 10\%). We also generate 400 simulated maps with the same lensing potential realizations as the original simulation set, but with different background $\mathrm{CMB}$ and noise realizations, which we use to calculate higher-order lensing biases (as first implemented in [37] and as described in the following section).

\section{LENSING PIPELINE}

In this section, we describe our method to estimate the CMB lensing power spectrum. The methodology in our pipeline is overall similar to that presented in $[20,23,26]$. 
Since a fixed projected dark matter map introduces statistical anisotropy into the CMB by gravitational lensing, $\mathrm{CMB}$ lensing introduces correlations between formerly independent Fourier modes of the CMB temperature and polarization fields. Exploiting these lensing-induced correlations between pairs of modes, we can reconstruct the lensing potential with quadratic estimators in the $\mathrm{CMB}$ temperature and polarization fields $X=\{T, E, B\}$ [38]:

$$
\bar{\phi}_{\mathbf{L}}^{X Y}=R_{\phi}^{X Y}(\mathbf{L}) \int \frac{d^{2} \mathbf{I}}{(2 \pi)^{2}} X(\mathbf{l}) Y(\mathbf{L}-\mathbf{l}) g_{\phi}^{X Y}(\mathbf{l}, \mathbf{L})
$$

where $g$ is a weighting function on the modes used in the quadratic estimators and $R_{\phi}$ is a normalizing function obtained analytically following [38]. The estimators we consider in our analysis are $X Y=\{T T, T E, E E, E B\}$, because the $T B$ estimator has negligible signal to noise and the $B B$ correlation is higher order. The two CMB maps $X, Y$ we use in the estimators have been filtered to include only scales $1000<|\mathbf{I}|<3000$. For a detailed discussion of this choice, focusing in particular on the minimum value of $|\mathbf{I}|$ used, see the Appendix. In addition, "stripes" of width $-90<\left|\mathbf{I}_{x}\right|<90$ and $-50<\left|\mathbf{I}_{y}\right|<50$ have been removed from the maps along Fourier axes corresponding to map declination and right ascension, respectively.

The function $g_{\phi}^{X Y}(\mathbf{I}, \mathbf{L})$ provides an optimal weighting given by the mean response of a pair of CMB fields $X(\mathbf{l}) Y(\mathbf{L}-\mathbf{l})$ to a lens $\phi_{\mathbf{L}}$, divided by the variance of this pair of fields. The simplest example is given by the $T T$ estimator, for which

$$
g_{\phi}^{T T}(\mathbf{l}, \mathbf{L})=\frac{C_{l}^{T T} \mathbf{l} \cdot \mathbf{L}+C_{|\mathbf{L}-\mathbf{l}|}^{T T} \mathbf{L} \cdot(\mathbf{L}-\mathbf{l})}{2\left(C_{l}^{T T}+N_{\mathbf{l}}^{T T}\right)\left(C_{|\mathbf{L}-\mathbf{l}|}^{T T}+N_{\mathbf{L}-\mathbf{l}}^{T T}\right)}
$$

where $C_{l}^{T T}$ is the temperature power spectrum including the peak smoothing from lensing and $N_{1}^{T T}$ is the temperature noise power spectral density. Analogous expressions for the other estimators can be found in [38], though we follow [39] and replace unlensed with lensed spectra in filters to cancel higher-order biases.

The normalization function $R_{\phi}^{X Y}(\mathbf{L})$ divides out the weights $g$ to ensure an unbiased estimator. As a first approximation, it is calculated analytically as in [38]. For example, for the $T T$ estimator, our first approximation to $R_{\phi}^{T T}$ is

$R_{\phi}^{T T}(\mathbf{L}) \approx L^{2}\left[\int \frac{d^{2} \mathbf{I}}{(2 \pi)^{2}} \frac{\left(g_{\phi}^{T T}(\mathbf{l}, \mathbf{L})\right)^{2}}{\left(C_{l}^{T T}+N_{\mathbf{l}}^{T T}\right)\left(C_{|\mathbf{L}-\mathbf{l}|}^{T T}+N_{\mathbf{L}-\mathbf{l}}^{T T}\right)}\right]^{-1}$.

We apply a small correction to this function when binning the estimator in $L$-space (e.g., to account for windowing effects); this correction is obtained by requiring that the cross-correlation of the reconstructed lensing field with the input lensing field from simulations recover the input lensing power spectrum of the simulations. In calculating this normalization correction, since two powers of the data mask enter into the quadratic lensing estimator, we apodize the input lensing potential simulations with the square of the data mask to mimic and absorb aliasing affecting the lensing reconstruction. For each estimator, the integrand of Eq. (1) can be written as sums of different convolutions of two Fourier space maps, so that the convolutions of Eq. (1) can be calculated using real space multiplications of different filtered fields [38]. The use of inverse FFTs in evaluating the integrals of Eq. (1) [and similarly, Eq. (3)] allows us to greatly speed up the lensing estimation and improve the scaling of computer time with map size. We assume the flat-sky approximation in our analysis, which is sufficiently accurate for the map sizes we use and the range of scales we seek to reconstruct $[14,26]$.

Even in the absence of lensing, anisotropic noise and window functions will produce spurious statistical anisotropy that affects the naive lensing estimator. An unbiased estimate of the potential can be recovered by subtracting this anisotropy signal, known as the mean field $\left\langle\bar{\phi}_{\mathbf{L}}^{X Y}\right\rangle$, that is induced by these types of nonlensing mode couplings. This mean field correction is calculated by averaging the reconstructions of the naive estimator from 400 simulations, each with independent $\mathrm{CMB}$ and lensing potential realizations. In this average, only the spurious, nonlensing mode couplings remain. We then recover an unbiased estimate of the lensing potential after subtracting this mean field:

$$
\hat{\phi}_{\mathbf{L}}^{X Y}=\bar{\phi}_{\mathbf{L}}^{X Y}-\left\langle\bar{\phi}_{\mathbf{L}}^{X Y}\right\rangle .
$$

We use barred variables to indicate biased estimators.

From these potential maps, we calculate the lensing power spectrum using the following naive estimator:

$$
\bar{C}_{L_{b}}^{\phi}[X Y, A B] \equiv \frac{1}{w_{4}} \sum_{b}\left\langle\hat{\phi}_{\mathbf{L}}^{X Y^{*}} \hat{\phi}_{\mathbf{L}}^{A B}\right\rangle
$$

where $X Y$ and $A B$ can be any of $T T, T E, E E, E B$, and the factor $w_{4}$ is calculated by taking each pixel value of the apodization mask to the fourth power and then averaging over pixels. To maximize the signal-to-noise ratio, the bandpowers are binned using a weight in the twodimensional Fourier plane $\mathbf{L}$ given by the fiducial signal and noise spectra using $\left(C_{L}^{\phi} /\left(C_{L}^{\phi}+R_{\phi}(\mathbf{L})\right)\right)^{2}$. Since each $\hat{\phi}_{\mathbf{L}}^{X Y}$ is a quadratic estimator in temperature and polarization, $\bar{C}_{L_{b}}^{\phi}$ is a four-point function in the CMB fields.

This naive lensing power spectrum estimate, Eq. (5), is biased, however, because a contribution to the lensing reconstruction power arises from both instrumental noise and the primary CMB. To obtain an unbiased estimator, this 
reconstruction "noise bias" must be subtracted. This bias can be understood if we consider averaging over the lensing field in addition to the background CMB; the measured power is comprised of a non-Gaussian (connected) part of the four-point function and a Gaussian (disconnected) part. The former is the lensing power spectrum of interest, and the latter must be subtracted off. We will refer to this bias term as the Gaussian bias (though it is often referred to as the " $N_{0}$ " bias).

In addition to the Gaussian bias, a bias must be subtracted that arises from additional connected contractions of two lensing potential fields in the measured fourpoint function, contributing at first order in the lensing power, and known as the " $N_{1}$ " bias. Furthermore, a small "Monte Carlo" (MC) bias must be simulated and subtracted to absorb any additional nonidealities not captured by the map-level mean field subtraction, for instance due to masking correlations beyond the mean field or higherorder corrections (we choose to treat this correction as additive, which is sufficient for small corrections). Finally, we also subtract a small modeled foreground bias $\Delta C_{L_{b}}^{F G}$ (3\% of the signal for temperature) from unresolved point sources and galaxy clusters, as detailed in Sec. V. For each temperature and polarization combination, after subtracting off the Gaussian, $N_{1}, \mathrm{MC}$, and foreground biases, the final unbiased estimate of the lensing power spectrum that we use for our analysis is given by [20,22]

$$
\begin{aligned}
\hat{C}_{L_{b}}^{\phi}[X Y, A B]= & \bar{C}_{L_{b}}^{\phi}[X Y, A B]-\Delta C_{L_{b}}^{\mathrm{Gauss}}[X Y, A B] \\
& -\Delta C_{L_{b}}^{N 1}[X Y, A B]-\Delta C_{L_{b}}^{\mathrm{MC}}[X Y, A B] \\
& -\Delta C_{L_{b}}^{\mathrm{FG}}[X Y, A B]
\end{aligned}
$$

where $\bar{C}_{L_{b}}^{\phi}$ is the biased estimate.

The biases we have described above are calculated as follows. The Gaussian $\left(N_{0}\right)$ bias is calculated using the method described by [37] from different pairings of data and simulation (superscript $S$ ) maps:

$$
\begin{aligned}
\Delta C_{L_{b}}^{\text {Gauss }}[X Y, A B]= & \left\langle\bar{C}_{L_{b}}^{\phi}\left[X Y^{S}, A B^{S}\right]+\bar{C}_{L_{b}}^{\phi}\left[X^{S} Y, A B^{S}\right]\right. \\
& +\bar{C}_{L_{b}}^{\phi}\left[X^{S} Y, A^{S} B\right]+\bar{C}_{L_{b}}^{\phi}\left[X Y^{S}, A^{S} B\right] \\
& -\bar{C}_{L_{b}}^{\phi}\left[X^{S} Y^{S^{\prime}}, A^{S} B^{S^{\prime}}\right] \\
& \left.-\bar{C}_{L_{b}}^{\phi}\left[X^{S} Y^{S^{\prime}}, A^{S^{\prime}} B^{S}\right]\right\rangle_{S, S^{\prime}}
\end{aligned}
$$

This method is constructed to self-correct for small differences between the two-point functions of simulations and data. This is accomplished by using the two-point correlation functions of the data, rather than the simulations alone, to calculate the Gaussian bias; the first four terms each isolate a different two-point contraction of data maps. The robustness to incorrect simulations can be seen in detail by expanding the two-point correlation function of the data about the two-point function of the simulation, and noting that the difference in this expression cancels to first order, as demonstrated in BICEP/Keck 2016 [21]. The detailed form of the estimator can be obtained by deriving an optimal trispectrum estimator from an Edgeworth expansion of the CMB likelihood [37]. Aside from providing robustness to simulations not matching real data, the use of a realization-dependent bias derived from data in the bias estimation has additional advantages such as reducing the correlation of different lensing potential bandpowers [39] as well as reducing the correlation of the lensing power spectra with the primary CMB spectra [40-42].

To calculate the average over simulations we use 100 different realizations of the simulation pairs $S, S^{\prime}$ to obtain this bias for each real and simulated measurement. We verify that increasing the number of different realizations from 50 to 100 did not substantially affect the results (with the amplitude only changing by $\sim 1 \%$ ). We therefore conclude that 50 realizations are sufficient for convergence, though we use 100 in our data to be conservative.

We obtain the $N_{1}$ bias by using simulations with different $\mathrm{CMB}$ realizations, but common lensing potential maps, following [20]:

$$
\begin{aligned}
\Delta C_{L_{b}}^{\mathrm{N} 1}[X Y, A B]= & \left\langle\bar{C}_{L_{b}}^{\phi}\left[X^{S_{\phi}} Y^{S_{\phi}^{\prime}}, A^{S_{\phi}} B_{\phi}^{S_{\phi}^{\prime}}\right]\right. \\
& +\bar{C}_{L_{b}}^{\phi}\left[X^{S_{\phi}} Y^{S_{\phi}^{\prime}}, A^{S_{\phi}^{\prime}} B^{S_{\phi}}\right] \\
& -\bar{C}_{L_{b}}^{\phi}\left[X^{S} Y^{S^{\prime}}, A^{S} B^{S^{\prime}}\right] \\
& \left.-\bar{C}_{L_{b}}^{\phi}\left[X^{S} Y^{S^{\prime}}, A^{S^{\prime}} B^{S}\right]\right\rangle_{S, S^{\prime}, S_{\phi}, S_{\phi}^{\prime}} .
\end{aligned}
$$

Here $S_{\phi}$ and $S_{\phi}^{\prime}$ indicate simulations with different CMB realizations but the same lensing potential realization, whereas $S$ and $S^{\prime}$ have different CMB and lensing potential realizations. Though subtracting the Gaussian and $N_{1}$ biases should result in a nearly unbiased lensing power spectrum, the Monte Carlo bias $C_{b}^{\mathrm{MC}}[X Y, A B]$ is obtained by calculating any remaining residual from simulations. We find this $\mathrm{MC}$ bias to be significantly smaller than our one sigma error, so we use it in our pipeline although its inclusion does not substantially change our results.

Finally, we combine our estimate of the lensing potential power spectrum for all three patches $p$ and all ten estimators $\alpha=\{T T, T T ; T T, T E ; T T, E E \cdots\}$ :

$$
\hat{C}_{L_{b}}^{\phi}=\sum_{\alpha, p} w_{\alpha, p, b} \hat{C}_{L_{b}}^{\phi, \alpha, p} .
$$

Here the weights for the bandpowers of each estimator and patch are given by the inverse of the variance of the bandpowers, obtained from 200 simulations. While our weights do not take into account correlation of the different estimators and maps, our final coadded error calculation does, because it simulates the full measurement and coadding procedure. We calculate error bars and a full covariance matrix for the final bandpowers by repeating the 


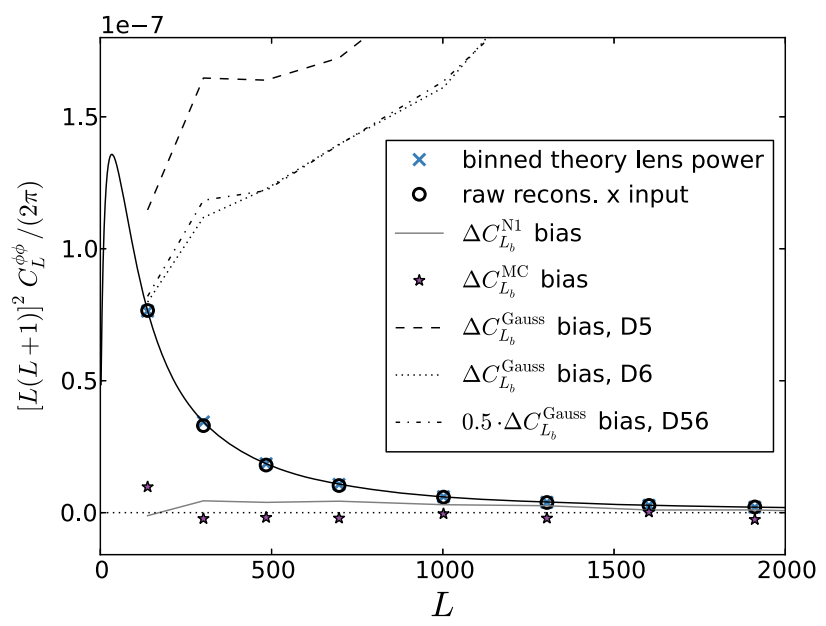

FIG. 1. Bias and signal terms from our pipeline simulations. The solid black circles show that the raw reconstructed maps correlate well with the input simulations (even without a simulation-based correction), closely matching the binned theory, denoted with light blue crosses. The dashed, dotted, and dotteddashed lines give the Gaussian $\left(N_{0}\right)$ bias for the D5, D6, and D56 patches respectively. The $N_{1}$ bias, indicated with a solid gray line, has a magnitude and shape consistent with expectations. The MC bias, indicated with stars, is small as expected. All curves are coadds over estimators and, where not indicated otherwise, over all patches, with the weights of Eq. (9). complete coadd power spectrum estimation procedure on 200 simulations. We discuss our systematic error estimate in Sec. $\mathrm{V}$ of this paper.

A plot showing the relevant bias terms for our pipeline, along with additional information useful for verification, is given in Fig. 1. Even before correcting the normalization function $R$ with simulations, we note that the crosscorrelation of the raw reconstructed lensing field with the input lensing field from simulations matches the input lensing power spectrum of the simulations to better than $5 \%$. In addition, we find the N1 bias to have the expected form and the MC bias to be small, which gives us further confidence.

\section{LENSING POWER SPECTRUM}

In Fig. 2, we show the final lensing power spectrum coadded over all estimators and patches, and in Table I we give the bandpower values and error bars. The amplitude of lensing power we obtain from the coadded result in Fig. 2, scaled from the Planck TT, TE, EE + lowP + lensing $\Lambda \mathrm{CDM}$ model of [23], is $A_{\text {lens }}$ of $1.06 \pm 0.15 \quad$ (stat) $\pm 0.06 \quad$ (sys). This represents a $7.1 \sigma$ measurement of the amplitude of lensing. Calculating a $\chi^{2}$ to our best-fit model, we obtain a probability to exceed (PTE) the given $\chi^{2}$ of 0.32 , indicating a

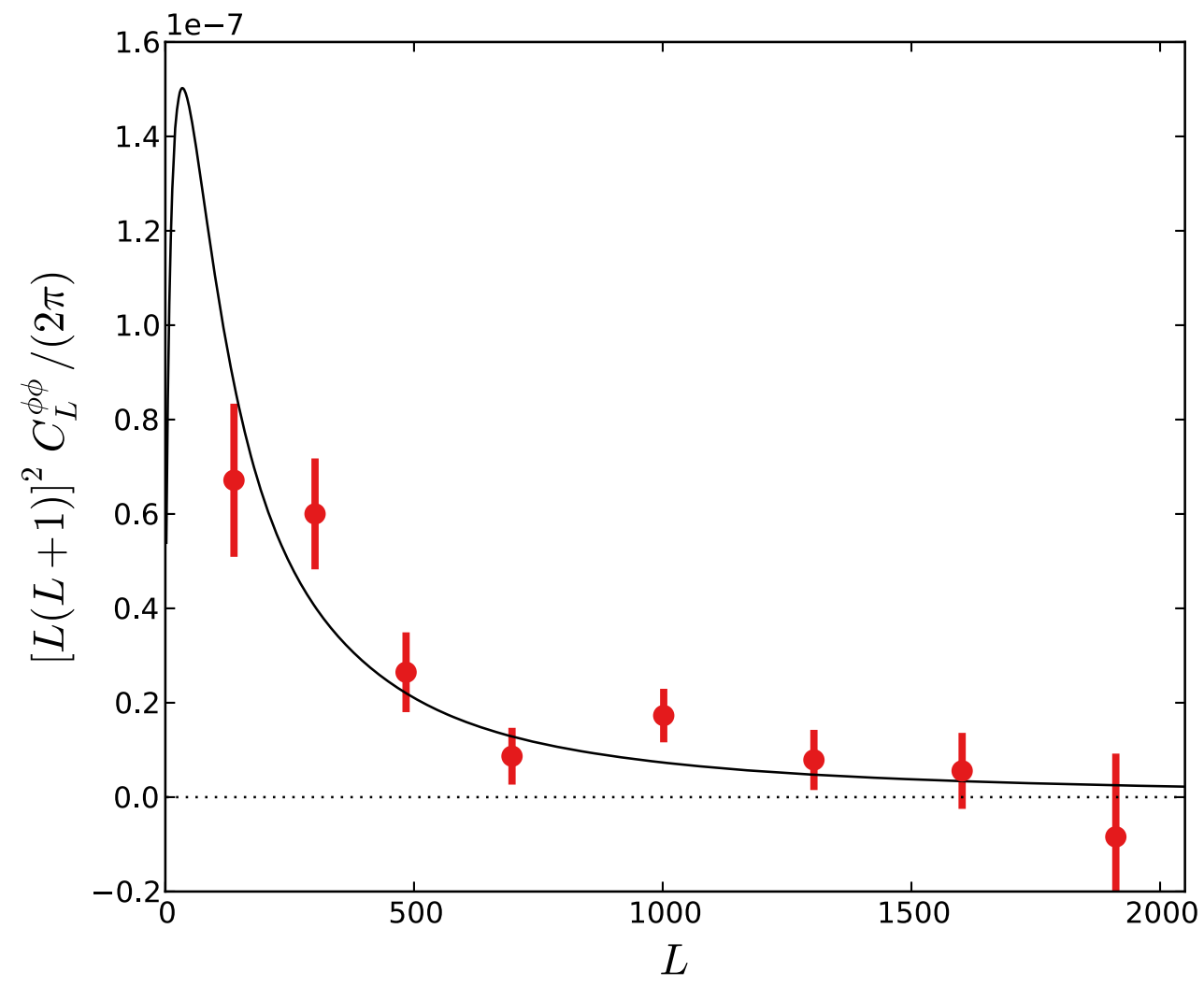

FIG. 2. Combined two-season ACTPol lensing power spectrum, coadded across all patches and estimators. The best-fit theory lensing power spectrum has an amplitude of $A_{\text {lens }}=1.06 \pm 0.15$ (stat) $\pm 0.06 \quad$ (sys) relative to the Planck best-fit $\Lambda$ CDM cosmology from the Planck temperature and polarization power spectra (which we define to have $A_{\text {lens }}=1$ ). The ACTPol best fit is indicated with a black solid line, and the error bars just include statistical uncertainty. The $\chi^{2}$ to the best-fit, scaled Planck $\Lambda$ CDM theory model has a probability to exceed (PTE) of 0.32 , suggesting a good fit to the standard $\Lambda$ CDM cosmology. 
TABLE I. ACTPol two-season lensing power spectrum bandpowers and $1 \sigma$ error bars.

\begin{tabular}{lcc}
\hline \hline$L$ & $L^{4} C_{L}^{\phi \phi} /\left(4 \times 10^{-7}\right)$ & $\sigma\left(L^{4} C_{L}^{\phi \phi}\right) /\left(4 \times 10^{-7}\right)$ \\
\hline 138 & 1.039 & 0.251 \\
301 & 0.937 & 0.183 \\
484 & 0.414 & 0.132 \\
697 & 0.136 & 0.094 \\
1002 & 0.271 & 0.089 \\
1304 & 0.124 & 0.100 \\
1602 & 0.087 & 0.126 \\
1911 & -0.132 & 0.277 \\
\hline \hline
\end{tabular}

good fit to $\Lambda \mathrm{CDM}$. This lensing amplitude is consistent with, and slightly higher than, that in the standard Planck cosmology.

In Fig. 3, we show our results broken down by estimator and patch. From Fig. 3, it can be seen that most of the constraining power comes from the temperature data in the wider D56 map. In Table II, we list the individual PTEs for the lensing power from each estimator and patch. Though there is one entry, the TE, TE estimator on D56, which has a low PTE of $0.44 \%$, we note that having a minimal PTE of this order is not unexpected, given that we calculate 30

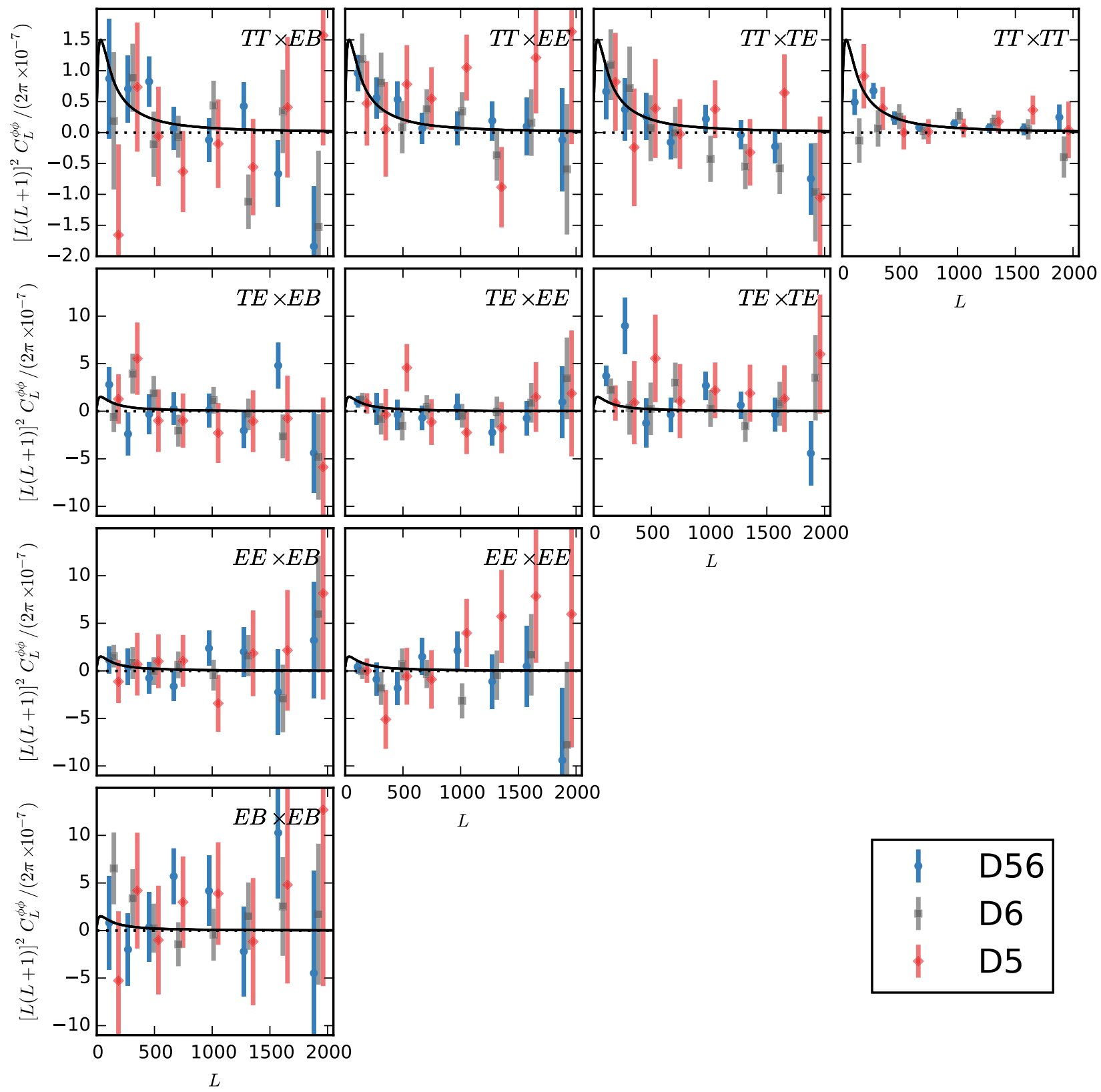

FIG. 3. Lensing power spectrum reconstructions for all the different patches and estimators separately. The blue points are from the D56 patch, the red from D5, and the gray from D6. We note that the fields overlap and are thus not independent. The black curve is the best-fit model. The $T T, T T$ measurement (top right) can be seen to dominate the combined result, but the other combinations with temperature (top row) also have significant weight. 
TABLE II. Probability to exceed the given $\chi^{2}$ for the lensing signal, compared to the best-fit model.

\begin{tabular}{lccc}
\hline \hline Estimator & D56 lens & D5 lens & D6 lens \\
\hline$T T, T T$ & 0.26 & 0.14 & 0.91 \\
$T E, T E$ & 0.004 & 0.74 & 0.94 \\
$E E, E E$ & 0.69 & 0.70 & 0.51 \\
$E B, E B$ & 0.42 & 0.84 & 0.94 \\
$T T, T E$ & 0.86 & 0.34 & 0.92 \\
$T T, E E$ & 0.92 & 0.79 & 0.22 \\
$T T, E B$ & 0.21 & 0.13 & 0.73 \\
$T E, E E$ & 0.84 & 0.88 & 0.64 \\
$T E, E B$ & 0.25 & 0.29 & 0.89 \\
$E E, E B$ & 0.77 & 0.92 & 0.92 \\
\hline \hline
\end{tabular}

signal PTEs and 30 null PTEs in this paper-in fact, a minimal PTE at or below this value occurs in $30 \%$ of our simulated measurements. Excluding the D56 TE, TE data shifts the best-fit overall $A_{\text {lens }}$ value downwards by only a small amount, approximately $0.25 \sigma$ (to $A_{\text {lens }}=1.02$ ).

\section{NULL TESTS AND SYSTEMATIC ESTIMATES}

We verify our results with null tests and targeted systematic checks. Neglecting very small corrections due to inadequacies of the lowest order Born approximation $[43,44]$, the lensing deflection field is given by the gradient of the lensing potential from scalar density perturbations. The deflection field hence is irrotational, with zero curl. However, a systematic that mimics lensing need not necessarily obey this gradientlike symmetry, and could hence also induce a curl-like deflection. Estimating the curl-like component of the deflection field yields a diagnostic for systematic errors that can mimic the lensing signal. We use a curl estimator given by

$$
\Omega_{\mathbf{L}}^{X Y}=R_{\Omega}^{X Y}(\mathbf{L}) \int \frac{d^{2} l}{(2 \pi)^{2}} X(\mathbf{l}) Y(\mathbf{L}-\mathbf{l}) g_{\Omega}^{X Y}(\mathbf{l}, \mathbf{L})
$$

where the filter $g_{\Omega}^{X Y}(\mathbf{l}, \mathbf{L})$ differs from the usual lensing estimation filter by the replacement of a dot product in the numerator with the perpendicular component of a crossproduct; the same modification occurs in the normalization function $R$. With this filter replacement, all the bias estimation steps are repeated in the same way as for the lensing estimation. The results for this null test are shown in Fig. 5 for each estimator and patch separately, and in Fig. 4 for the coadded result. The curl PTEs for each estimator and patch with respect to zero are shown in Table III, and are consistent with zero. For the coadded curl, the PTE with respect to zero is 0.57 , a good agreement with null.

We also investigate the stability of our lensing power spectrum measurement to specific sources of systematic error. In Fig. 6, we show our measurement of the lensing power spectrum repeated with maps that have been

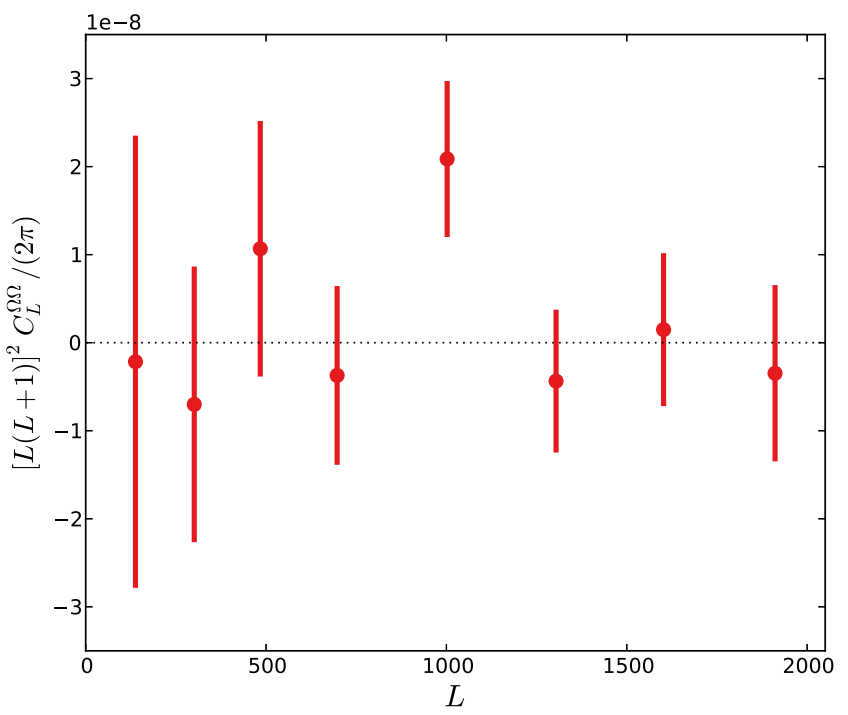

FIG. 4. Curl null test, coadded over all patches and estimators with the same weights as the lensing potential power spectrum. The PTE with respect to zero is 0.57 .

perturbed by realistic levels of different sources of instrumental or astrophysical error. The sources of error we consider are described in the following paragraphs. For each potential systematic effect, we note the change in the best-fit lensing amplitude $A_{\text {lens }}$, yielding an approximate estimate of its contribution to the total systematic error on our measurement.

\section{A. Beam uncertainty}

We vary the beam within the uncertainties given in L16, coherently perturbing the beams in both temperature and polarization for all patches upwards by one standard deviation in order to obtain a conservative estimate. As shown in Fig. 6, we find only small changes in the lensing bandpowers and a negligible overall shift of $\Delta A_{\text {lens }}<0.01$.

\section{B. Calibration uncertainty}

We show the impact of CMB calibration uncertainty in Fig. 6. As the limits quoted by L16 are $\approx 1 \%$, the bandpowers are perturbed by a factor $\approx 1.04$. The corresponding shift in the lensing amplitude is similarly $\Delta A_{\text {lens }}=0.04$. We include this error as a contribution to the total systematic error on $A_{\text {lens }}$.

\section{Polarization angle uncertainty}

We model a global polarization angle offset within the stated limits of $\mathrm{L} 16$ by adding $1 \%$ of the $Q$ maps to $U$ and subtracting $1 \%$ of the $U$ maps from $Q$. This results in small shifts to lensing bandpowers and a change in the overall amplitude of $\Delta A_{\text {lens }}=0.01$. We again include this value in our total systematic error budget. 


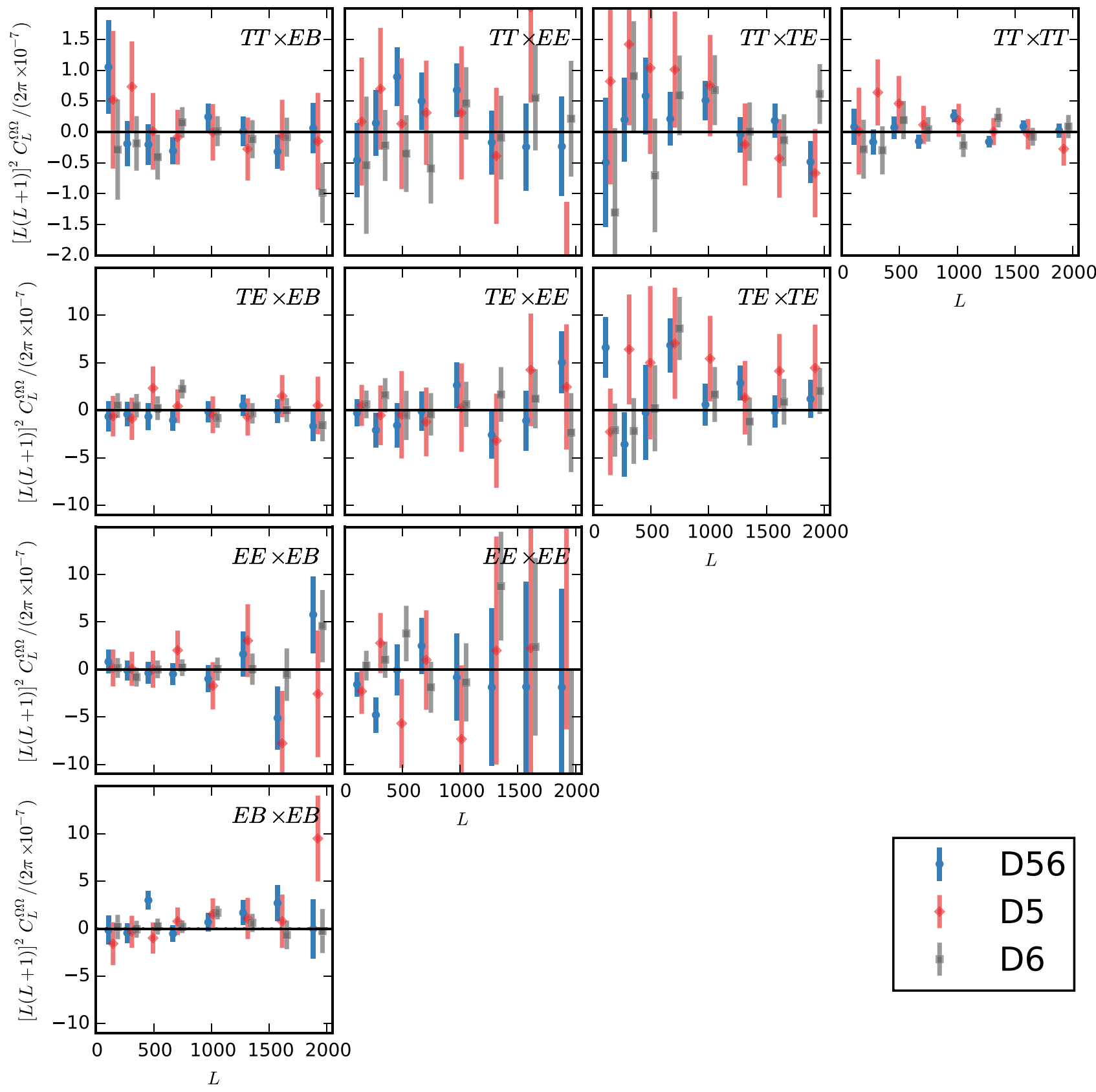

FIG. 5. Curl null test power spectra for all the different patches and estimators separately. The blue points are from D56, the red points are from D5, and the gray points are from D6.

\section{Temperature-to-polarization leakage}

We model instrumental temperature-to-polarization leakage by adding $1 \%$ of the temperature map to the $E$-mode map (as a leakage of this form and magnitude was found to be present in initial versions of the ACTPol CMB maps, though it was fixed by better beam characterization, as described in L16). We again find only small shifts to bandpowers and a change in the amplitude of lensing of $\Delta A_{\text {lens }}=-0.02$, which we include in our total systematic error calculation.

\section{E. Galactic dust}

We calculate an upper bound on the impact of galactic dust by subtracting the Planck $353 \mathrm{GHz}$ maps below $\ell<$ 2000 from our CMB temperature maps (at higher $\ell$, cosmic infrared background (CIB) and instrumental noise become large and dominant). Prior to subtraction, we rescale the $353 \mathrm{GHz}$ maps to serve as dust maps at $149 \mathrm{GHz}$ by dividing by $\approx 20$ (see [23]). We obtain a shift of $\Delta A_{\text {lens }}=-0.03$, with only small changes in the lensing bandpowers. Though this value represents in some sense an 
TABLE III. Probability to exceed the given $\chi^{2}$ for the curl signal, compared to null.

\begin{tabular}{lccc}
\hline \hline Estimator & D56 curl & D5 curl & D6 curl \\
\hline$T T, T T$ & 0.10 & 0.89 & 0.60 \\
$T E, T E$ & 0.05 & 0.66 & 0.29 \\
$E E, E E$ & 0.43 & 0.74 & 0.58 \\
$E B, E B$ & 0.08 & 0.50 & 0.55 \\
$T T, T E$ & 0.49 & 0.75 & 0.45 \\
$T T, E E$ & 0.36 & 0.64 & 0.96 \\
$T T, E B$ & 0.53 & 0.99 & 0.54 \\
$T E, E E$ & 0.51 & 0.995 & 0.99 \\
$T E, E B$ & 0.90 & 0.98 & 0.43 \\
$E E, E B$ & 0.51 & 0.82 & 0.94 \\
\hline \hline
\end{tabular}

upper bound (since a small fraction of the large-scale CMB is also removed), we include this value in our systematic error budget. Comparable small bounds were found in $[16,23]$. We note that the impact of polarized dust is expected to be very small, given that we only use information at $\ell>1000$ and given that most of our statistical weight is in the temperature estimator. Furthermore, we note that the curl null should be sensitive to an unexpectedly large dust bias [20].

\section{F. Source and cluster mask level and mask size}

Steps have been taken in this analysis to mitigate the impact of astrophysical contaminants, such as observing in low-dust regions, masking and in-painting SZ clusters, and template-subtracting bright star-forming and radio galaxies. However, we also test for any effect on our results from residual astrophysical foregrounds. In Fig. 6, we show the result of changing the number of masked clusters and residual sources, with mask thresholds corresponding to objects detected at $6 \sigma, 5 \sigma$, and $4 \sigma$ using a matched filter. Our main result masks out SZ clusters and residual sources above $5 \sigma$. The variation in bandpowers and in the amplitude of lensing is much less than the statistical error for all masking choices, with a root-mean-squared change of $\Delta A_{\text {lens }}=0.03$ from the baseline result. We further test the stability of our results by doubling the size of the inpainting mask around each object. We find only small changes to bandpowers and an overall shift of $\Delta A_{\text {lens }}=-0.01$. Finally, we display in Fig. 6 the lensing bandpowers when no masking of clusters and residual sources is performed. As expected, omitting the masking procedure entirely causes substantial shifts in the bandpowers. However, as shown in Fig. 6 our results are insensitive to the details of the masking procedure, which gives confidence in their robustness.

\section{G. Unresolved astrophysical foregrounds}

Even with aggressive masking, some residual effective lensing signal will remain from the trispectra associated
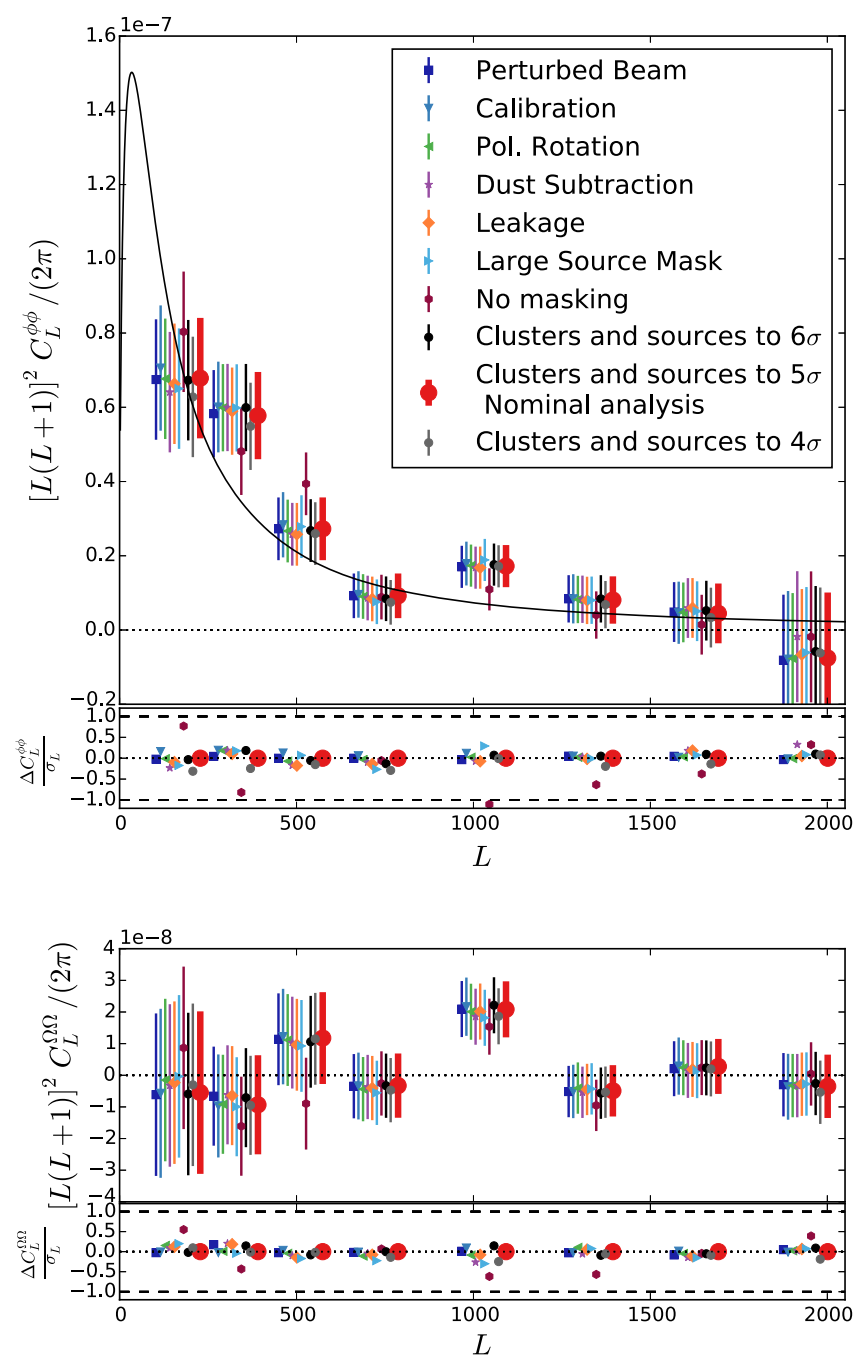

FIG. 6. Systematic test summary plot. The top panel shows the stability of our results to realistic levels of potential sources of systematic error, including instrumental errors in beams, calibration, polarization angle and temperature to polarization leakage, as well as astrophysical systematics such as galactic dust (see text for details). We also show the stability of our results when changing the masking threshold for SZ clusters and irregular point sources. The second panel shows the same data, but represented in terms of standard deviations. We note the stability of the results to realistic levels of instrumental systematic effects and analysis choices (though it is apparent that some degree of source masking is required). The bottom two panels show the equivalent points for the curl null test.

with extragalactic objects just below the cut threshold in the temperature maps [45]. These biases, arising from galaxy clusters, the cosmic infrared background, and radio sources, were estimated in [46], based partly on the simulations from [47]. We find that for our current masking levels and maximum multipole used in the reconstructions, the biases expected are roughly $3 \%$ of the signal for the $T T, T T$ estimator. We use the relevant curves from [46] as our foreground bias $\Delta C_{L_{b}}^{F G}$ that we subtract when deriving 
our final lensing power spectrum. For polarization, the foregrounds are expected to be much less of a concern-SZ clusters produce only an extremely small polarized signal, and the polarized CIB and point source levels are also very small (e.g., L16). We thus neglect unresolved foreground biases in estimators using only polarization. For lensing power spectrum estimators involving one $T T$-estimator half (e.g, $T T, E B$ ), we assume a bias $\Delta C_{L_{b}}^{F G}$ given by one half of the $T T, T T$ bias (which is justified by a dominant contribution to the bias arising from the lensing-source-source bispectrum [46]). If we turn off subtraction of the entire bias, the amplitude of lensing shifts by $\Delta A_{\text {lens }}=-0.02$.

What contribution from astrophysical foregrounds such as galaxy clusters, CIB, and radio sources should we assign to our overall systematic error budget? We note that there is of order $50 \%$ theoretical uncertainty on the simulationderived estimate [46], implying an error $\Delta A_{\text {lens }} \approx 0.01$. To be conservative, we add (in quadrature) to this the dispersion found for different source and cluster masking levels, giving a total error of $\Delta A_{\text {lens }}=0.03$ for the astrophysical uncertainty in our measurement.

\section{H. Noise tests}

Finally, we test our modeling of the noise. By differencing two splits of our data with equal weight and thus canceling the signal, we obtain maps of the noise in our data. We add these maps to simulations of the lensed CMB signal and measure the lensing power spectrum of the resulting maps with our pipeline. The recovered lensing power spectrum is found to be a good fit to the input simulation power spectrum, with a PTE of $76 \%$. We repeat this analysis with a new realization of the background CMB signal, obtaining a PTE of $6 \%$. From different splits of our data, we also obtain a new, uncorrelated noise (and signal) map, which gives a PTE of $75 \%$ in our test. For all three cases, the 30 individual PTEs for each patch and estimator combination appear nominal. We therefore find no significant evidence for systematics from noise modeling in our analysis.

In Fig. 6, we also show the changes to the curl null test in response to the enumerated systematic effects. As none of the systematics or analysis choices we investigate (aside from not masking any sources at all) causes significant changes to the curl points, we conclude that the systematic effects investigated are not responsible for any features in the curl power spectrum.

We summarize the different sources of systematic error investigated, along with an approximate (often conservative) estimate of their impact on the amplitude of lensing, $\Delta A_{\text {lens }}$, in Table IV. By adding all these sources of error in quadrature, we obtain an estimate for the total systematic error on our measurement of the lensing power spectrum amplitude of $\Delta A_{\text {lens }}$ (sys) $=0.06$.

For the systematic tests enumerated above, we note that the overall systematic error contribution is subdominant to
TABLE IV. Systematic error budget. We list the different sources of systematic error investigated, along with an approximate (often conservative) estimate of their impact on the amplitude of lensing, $\Delta A_{\text {lens }}$. Adding the different errors in quadrature, we obtain an estimate for our total systematic error, $\Delta A_{\text {lens }} \quad$ (sys).

\begin{tabular}{lc}
\hline Type of systematic & Systematic error, $\Delta A_{\text {lens }}$ \\
\hline Beams & $<0.01$ \\
Calibration & 0.04 \\
Polarization angle & 0.01 \\
Temperature-polarization leakage & 0.02 \\
Galactic dust & $<0.03$ \\
Astrophysical (clusters/sources) & 0.03 \\
Total systematic error & 0.06 \\
\hline \hline
\end{tabular}

the statistical error. In all tests, we do not see significant changes to our baseline results. Indeed, nearly all our estimates of systematics are conservative upper limits; there is no significant evidence for systematic contamination to our lensing measurement at the current level of precision from either astrophysical or instrumental effects.

\section{COSMOLOGICAL PARAMETERS}

In this section, we present cosmological constraints on the linear-theory matter fluctuation amplitude $\sigma_{8}$, the matter density $\Omega_{m}$, and the sum of the neutrino masses $\Sigma m_{\nu}$ from the ACTPol lensing power spectrum. We obtain these constraints from the coadded lensing power spectrum shown in Fig. 2.

We model the ACTPol lensing likelihood by assuming Gaussian uncertainties on the correlated, binned coadded spectrum, $\hat{C}_{L_{b}}^{\phi}$, so that the log-likelihood is given by

$-2 \ln \mathcal{L}=\sum_{b b^{\prime}}\left[\hat{C}_{L_{b}}^{\phi}-C_{L_{b}}^{\phi, \text { th }}(\boldsymbol{\theta})\right] \mathbb{C}_{b b^{\prime}}^{-1}\left[\hat{C}_{L_{b^{\prime}}}^{\phi}-C_{L_{b^{\prime}}}^{\phi, \text { th }}(\boldsymbol{\theta})\right]$.

The Gaussian approximation is justified by the large number of effective independent modes in our bandpowers. We have checked that a correction due to having a finite number of simulations, based on [48], yields only a 2-3\% effect on our final bandpower errors. The covariance matrix $\mathbb{C}^{-1}$ for the binned spectrum is calculated using Monte Carlo simulations as described in Sec. III. Since the normalization $R_{\phi}(L)$ in Eq. (1) and the $\Delta C_{L_{b}}^{\mathrm{N} 1}$ bias correction in Eq. (8) assume a fiducial cosmology $\boldsymbol{\theta}_{0}$, we calculate the expected spectrum, $C_{L}^{\phi, \text { th }}(\boldsymbol{\theta})$, at the point $\boldsymbol{\theta}$ in cosmological parameter space and correct it to reflect the $R_{\phi}(L)$ and $C_{L_{b}}^{\mathrm{N} 1}$ we used for the data. Since calculating the exact correction for each point in parameter space is prohibitively slow, we follow the approach in [23] and exploit the near-linear dependence of the expected power spectrum, due to shifts in $R_{\phi}(L)$ and $\Delta C_{L_{b}}^{\mathrm{N} 1}$, when expanding around the fiducial cosmology [see in particular 
Eq. (C.5) in [23]]. However, we neglect the contribution to the correction from the dependence of $\Delta C_{L_{b}}^{\mathrm{N} 1}$ on the CMB primordial power spectra as these spectra are strongly constrained by the addition of CMB power spectrum information. In addition, the dependence of $\Delta C_{L_{b}}^{\mathrm{N} 1}$ on the lensing power spectrum is assumed to be dominated by an overall scaling of the amplitude of the fiducial lensing power spectrum rather than on scaling each $L_{b}$ mode separately; this is a very good approximation for the parameters we consider, which effectively only smoothly rescale the lensing power spectrum. For any pair of estimators $X Y, A B$ used for the power spectrum we therefore have

$$
\begin{aligned}
C_{L, X Y A B}^{\phi, \mathrm{th}}= & C_{L}^{\phi}+\left.\frac{d \ln R_{\phi}^{X Y} R_{\phi}^{A B}(L)}{d C_{\ell}^{j}}\right|_{\boldsymbol{\theta}_{0}}\left(C_{\ell}^{j}(\boldsymbol{\theta})\right. \\
& \left.-C_{\ell}^{j}\left(\boldsymbol{\theta}_{0}\right)\right) C_{L}^{\phi}\left(\boldsymbol{\theta}_{0}\right) \\
& +\Delta C_{L, X Y A B}^{\mathrm{N} 1}\left(\boldsymbol{\theta}_{0}\right)\left(\frac{\left\langle C_{L}^{\phi}(\boldsymbol{\theta})\right\rangle}{\left\langle C_{L}^{\phi}\left(\boldsymbol{\theta}_{0}\right)\right\rangle}-1\right),
\end{aligned}
$$

where $C_{L}^{\phi}$ is the theory power spectrum for the given parameters, and where we estimate the mean amplitude of lensing $\left\langle C_{L}^{\phi}(\boldsymbol{\theta})\right\rangle$ by averaging $L$ times the lensing convergence power $\left(\sim L^{5} C_{L}^{\phi}\right)$ from $L=0-2000$.

The final theory lensing spectrum that is compared against the measured coadded lensing spectrum is the linear combination of the above spectra over all $X Y, A B$ pairs, weighted and binned in the same way as the measured coadded lensing spectrum [Eq. (5)].

We calculate theory power spectra using the Boltzmann code CAMB (using Halofit to model the effects of nonlinear structure formation [49,50]) and use the MCMC code CosmoMC [51,52] to obtain parameter constraints. We consider the basic six $\Lambda$ CDM parameters-cold dark matter and baryon densities $\Omega_{c} h^{2}$ and $\Omega_{b} h^{2}$, the optical depth to reionization $\tau$, the Hubble constant $H_{0}$, and the amplitude and scalar spectral index of primordial fluctuations $A_{s}$ and $n_{s}$ - and a single family of massive neutrinos with total mass $\Sigma m_{\nu}$. These parameters are varied with priors as summarized in Table $\mathrm{V}$ and consistently with the Planck lensing analysis [23]. We, however, update the $\tau$ estimate following more recent Planck data [53]. The prior on $\Omega_{b} h^{2}$ comes from big bang nucleosynthesis in combination with quasar absorption line observations [54], and the prior on $n_{s}$ is centered on Planck measurements of the CMB power spectra but with a relatively broad width [55].

As explained in detail in [23], the parameter combination that lensing measures best is $\sigma_{8} \Omega_{m}^{0.25}$. From ACTPol lensing alone, we obtain a constraint in the $\sigma_{8}-\Omega_{m}$ plane of

$$
\sigma_{8} \Omega_{m}^{0.25}=0.643 \pm 0.054 \quad(\text { ACTPollens only, 68\%) }
$$

TABLE V. Priors used in the cosmological analysis when including and not including primary $\mathrm{CMB}$ temperature fluctuations.

\begin{tabular}{lcc}
\hline \hline Parameter & Without CMB $T T$ & With CMB $T T$ \\
\hline $\ln 10^{10} A_{s}$ & {$[2,4]$} & {$[2,4]$} \\
$H_{0}$ & {$[40,100]$} & {$[40,100]$} \\
$n_{s}$ & $0.96 \pm 0.02$ & {$[0.8,1.2]$} \\
$\Omega_{b} h^{2}$ & $0.0223 \pm 0.0009$ & {$[0.005,0.1]$} \\
$\Omega_{c} h^{2}$ & {$[0.005,0.99]$} & {$[0.005,0.99]$} \\
$\tau$ & $0.058 \pm 0.012$ & $0.058 \pm 0.012$ \\
$\sum m_{\nu}(\mathrm{eV})$ & 0.06 & {$[0,10]$} \\
\hline \hline
\end{tabular}

This is consistent with the Planck lensing-only constraint of $\sigma_{8} \Omega_{m}^{0.25}=0.591 \pm 0.021$ [23]. Though the Planck lensing power spectrum measurement itself is much more precise than our measurement, the constraints on $\sigma_{8} \Omega_{m}^{0.25}$ are more comparable, because Planck's constraint on the $\sigma_{8} \Omega_{m}^{0.25}$ combination is degraded by marginalizing over $\Omega_{m} h^{2}$ and other parameters.

Combining the ACTPol lensing likelihood with a baryon acoustic oscillations (BAO) likelihood, which includes 6DF [56], SDSS MGS [57], and BOSS DR12 CMASS and LOWZ data sets [58], we break the $\sigma_{8}-\Omega_{m}$ degeneracy and obtain the following individual marginalized constraints,

$\sigma_{8}=0.8321 \pm 0.053 \quad($ ACTPollens $+\mathrm{BAO}, 68 \%)$

$\Omega_{8}=0.418 \pm 0.042 \quad$ (ACTPollens $\left.+\mathrm{BAO}, 68 \%\right)$.

We note that the constraints given in Eqs. (13)-(15) are obtained while fixing the cosmology $\boldsymbol{\theta}$ in the $R_{\phi}(L)$ correction given in Eq. (12) to the Planck best-fit model from the Planck primary CMB data alone, just as done for the Planck lensing-only constraints obtained in [23]. This restricts the statistics of the CMB background source light, giving a weaker constraint than fully adding the Planck primary $\mathrm{CMB}$ data to the ACTPol and BAO data sets. If we allow the cosmology in the $R_{\phi}(L)$ correction to vary when only ACTPol lensing data are used, then the parameter chains explore regions of parameter space that are largely inconsistent with known measurements of the primary $\mathrm{CMB}$, due to a degeneracy of the amplitude of the lensing signal with the $\mathrm{CMB}$ power spectra [in our case, primarily with an integral scaling as $\left.\left(C_{\ell}^{T T}\right)^{2}\right]$.

We present our constraints in Fig. 7. The red contours show the ACTPol lensing-only results with the source plane fixed in the $R_{\phi}(L)$ correction to the best-fit Planck primary CMB cosmology. The blue contours show the result when adding $\mathrm{BAO}$, again fixing $\boldsymbol{\theta}$ in the $R_{\phi}(L)$ correction to the Planck best-fit model. We compare with the corresponding Planck lensing plus BAO contours shown in gray. BAO alone has a mild preference for $\Omega_{m} \approx$ 0.4 in this plane, and it intersects the ACTPol-only contours around this value. However, in the $H_{0}-\Omega_{m}$ plane, there is only a small parameter region where $\mathrm{BAO}$ and Planck lens 


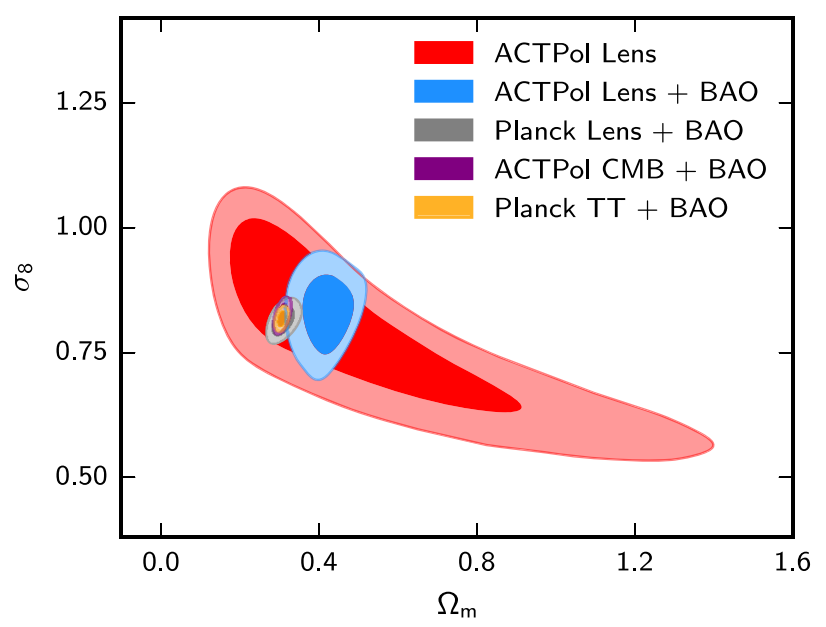

FIG. 7. Constraints at $68 \%$ C.L. on $\sigma_{8}$ versus $\Omega_{m}$ from ACTPol lensing data alone and in combination with $\mathrm{BAO}$ data. The red contours are from ACTPol lensing data (i.e. four-point correlation function information) alone when fixing the cosmology used in the lensing power spectrum normalization correction to the best fit from Planck primary CMB data; this effectively restricts the background source plane to be consistent with primary $\mathrm{CMB}$ data. The blue contour shows the result when BAO data are added (see text for details of the data set). Planck four-point lensing data plus BAO are indicated by the gray contour. For comparison with high redshift probes of the amplitude of structure, which do not necessarily probe the same physics in extensions to $\Lambda \mathrm{CDM}$, we also show the contours from the CMB power spectra, for both ACTPol and Planck data plus BAO, as purple and yellow contours, respectively. These CMB power spectrum contours, which mainly probe the primary $\mathrm{CMB}$, are consistent with our lower redshift lensing measurements.

contours intersect, which is around $\Omega_{m} \approx 0.3$. Thus, the gray Planck lens plus BAO contour is centered around $\Omega_{m} \approx 0.3$, though the reason for this is not immediately apparent from the $\sigma_{8}-\Omega_{m}$ plane alone.

In Fig. 7, we also show Planck primary $T T$ plus BAO and ACTPol primary CMB plus BAO constraints. CMB power spectrum measurements give a measurement of lensing through peak smoothing of the primary spectrum. We call this lensing measurement "two-point lensing," in contrast to the lensing power spectrum measurement discussed in this work, which we call "four-point lensing." We note that the Planck and ACTPol primary CMB measurements plus $\mathrm{BAO}$ are very constraining, both due to their measurements of the two-point lensing signal and because they constrain the amplitude of high-redshift structure via the optical depth $\tau$.

In Fig. 8, we show a compilation of recent CMB lensingonly (four-point) and optical-lensing only constraints. The optical lensing constraints are from CFHTLens [59], KiDS [60] and DES [61], and are derived from measurements of galaxy shapes that have been distorted by lensing from intervening matter. The DES chains provided by the DES team only extend to $\Omega_{m} \simeq 0.9$. This plot shows consistency between the data sets given their uncertainties.

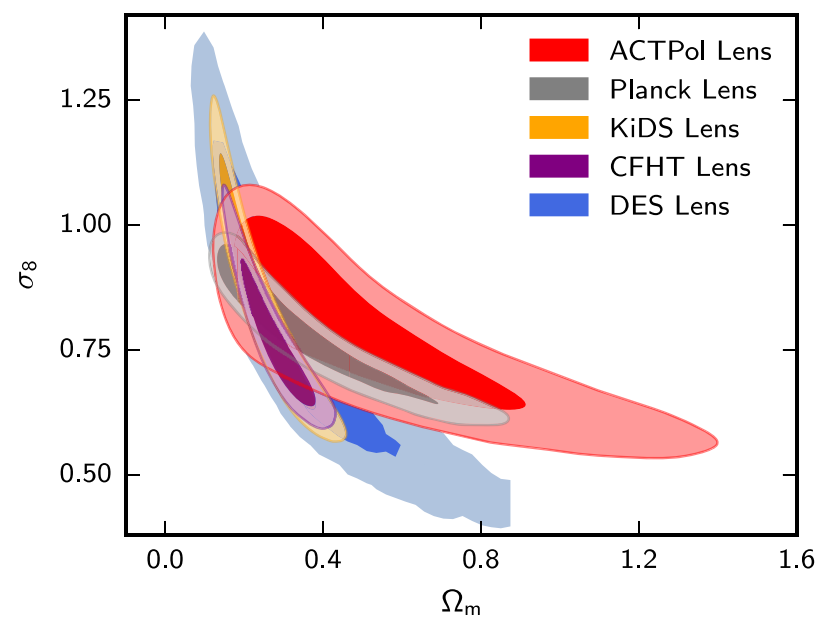

FIG. 8. Compilation of recent constraints on $\sigma_{8}$ versus $\Omega_{m}$ from $\mathrm{CMB}$ and optical lensing measurements (CFHTLens [59], KiDS [60] and DES [61]). The CMB constraints are from the lensing power spectrum information only. The data sets are seen to be broadly consistent, and the degeneracy direction from the CMB experiments can be seen to differ from those from the optical surveys.

The presence of massive neutrinos suppresses the growth of structure and thus reduces the amplitude of lensing. In the $\mathrm{CMB}$, neutrino mass constraints therefore arise via a constraint on the lensing amplitude from four-point lensing (the lensing power spectrum) or from two-point lensing (the $\mathrm{CMB}$ power spectrum peak smoothing). Within $\nu$ LCDM, the amplitude of both effects (and their ratio) is predicted uniquely. If their amplitude differs significantly in the data, caution may be called for in the interpretation of one or both of the measurements unambiguously as a lensing signal $[62,63]$.

To constrain the sum of neutrino masses, we combine our lensing measurement with the ACTPol two-season CMB temperature and polarization power spectra [30], and with BAO. Since our lensing maps are nearly noise dominated and since we use a data-dependent Gaussian bias subtraction, we can neglect the covariance of the lensing and CMB power spectrum measurements [40]. With this combination, we obtain a constraint of

$$
\begin{aligned}
\Sigma m_{\nu}< & 0.396 \mathrm{eV} \quad(\text { ACTPol lens } \\
& + \text { ACTPol CMB + BAO, 95\%) } .
\end{aligned}
$$

For this result, the cosmology in the $R_{\phi}(L)$ correction was allowed to be free. We show this constraint as the thick red curve in Fig. 9.

In Fig. 9, we also show the constraint combining Planck four-point lensing plus Planck primary CMB TT (the Planck temperature power spectrum at all scales) plus $\mathrm{BAO}$, as the solid blue curve. This constraint is $\Sigma m_{\nu}<0.153 \mathrm{eV}$ at 95\% C.L., which is somewhat tighter than reported in [23]. This tightening of the $\Sigma m_{\nu}$ constraint is due to the use of DR12 as opposed to DR11 when including BOSS BAO, and the 


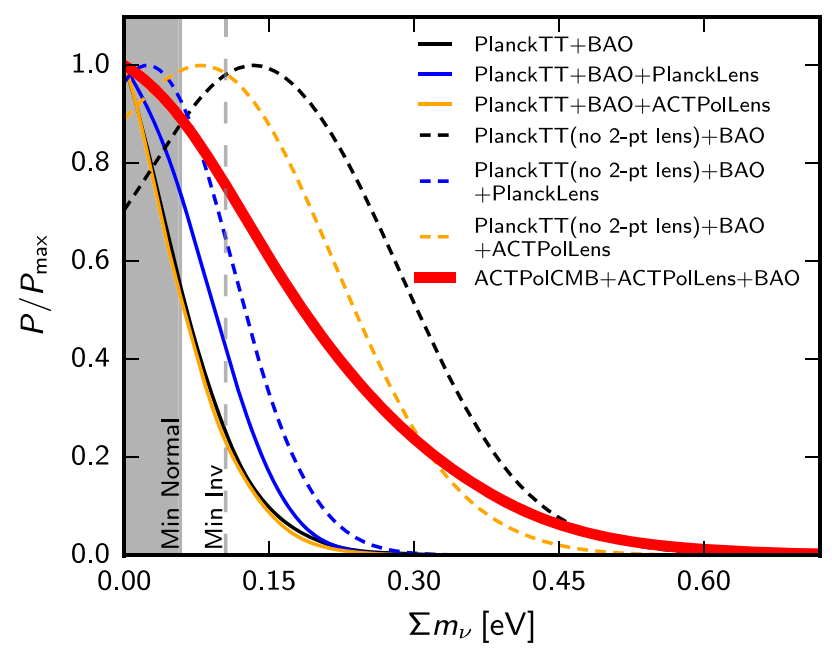

FIG. 9. Constraint on neutrino mass marginalized over the other parameters from ACTPol primary $\mathrm{CMB}+\mathrm{ACTPol}$ four-point lensing + BAO (thick red curve). We also show Planck TT + BAO (black solid curve), Planck TT + BAO + Planck four-point lens (blue solid curve), and Planck TT + BAO + ACTPol fourpoint lens (gold solid curve). The black dashed curve shows Planck $T T+$ BAO when we remove the two-point lensing signal from peak smoothing in Planck TT (see details in text), and the gold dashed curve shows the result when ACTPol four-point lensing is added to that. The difference between the black and gold dashed curves isolates the improvement when adding ACTPol four-point lensing data, and comparison of the gold dashed and solid curves shows the effect from adding the two-point lensing information in Planck TT. We also show the minimal neutrino masses for the normal and inverted hierarchy of 58 and $105 \mathrm{meV}$, respectively, assuming that the cosmological neutrinos have the same properties as those measured in terrestrial experiments [64].

lower central value and tighter error bar on $\tau, 0.058 \pm 0.012$ versus $0.067 \pm 0.017$, that was recently reported in [53].

The constraint without Planck four-point lensing, i.e. from Planck TT and BAO alone, is shown as the solid black curve. Omitting the Planck four-point lensing actually makes the constraint slightly stronger, because the large two-point peak smoothing is mildly discrepant with the four-point lensing amplitude.

To explore constraints on the neutrino mass which do not rely on two-point smoothing of the CMB $T T$ spectrum and to quantify the constraining power of current ACTPol lensing, we free the parameter $A_{\text {lens }}$, allowing it to vary just the two-point peak smoothing in the Planck TT spectrum [65]. Marginalizing over $A_{\text {lens }}$ effectively removes the peaksmoothing information from the Planck two-point TT measurement. The resulting constraints are shown as dashed lines in Fig. 9. The black dashed curve shows Planck $T T$ and BAO alone, marginalizing over $A_{\text {lens }}$; the gold and blue dashed curves include ACTpol and Planck lensing measurements. The two-point data and BAO (black dashed) give a mass constraint of $\Sigma m_{\nu}<0.378 \mathrm{eV}$ at 95\% C.L. Adding ACTPol four-point lensing (the gold dashed curve) yields a constraint of $\Sigma m_{\nu}<0.320$ at
95\% C.L. This improvement is from the ACTPol fourpoint lensing measurement alone.

\section{CONCLUSIONS}

We report a new measurement of the power spectrum of CMB lensing from two seasons of ACTPol CMB temperature and polarization data. This measurement can be compared with those of other groups in Fig. 11. We detect lensing power at high significance in our data and find the lensing power spectrum to be consistent with $\Lambda \mathrm{CDM}$ predictions. No evidence for significant systematic effects is seen in our null tests and checks. We obtain an amplitude of lensing power $A_{\text {lens }}=1.06 \pm 0.15$ (stat) \pm 0.06 (sys), a $7.1 \sigma$ measurement, and an amplitude of density fluctuations $\sigma_{8}=0.831 \pm 0.053$. Both measurements are consistent with the Planck $\Lambda \mathrm{CDM}$ cosmology (which we define to have $A_{\text {lens }}=1$ ). While the amplitude of density fluctuations we report is higher than that found in some recent weak lensing surveys [24], our uncertainties are currently still too large to resolve any claimed tensions between Planck and these low-redshift tracers. However, we note that our current measurements are based on only $12 \%$ of the ACTPol observational data [30]. As the remaining ACTPol data are included in our analysis, using the pipeline described in detail in this paper, we expect to report significantly improved measurements of the lensing power spectrum. This will, in turn, give stronger constraints on the amplitude of structure and on cosmological parameters such as the neutrino mass.

\section{ACKNOWLEDGMENTS}

The authors would like to thank Anthony Challinor, Antony Lewis, and Toshiya Namikawa for useful discussions, and are grateful to Kyle Story for comments on the draft. This work was supported by the U.S. National Science Foundation (NSF) through Grants. No. AST-1440226, No. AST-0965625 and No. AST-0408698 for the ACT project, as well as Grants No. PHY-1214379 and No. PHY-0855887. Funding was also provided by Princeton University, the University of Pennsylvania, and a Canada Foundation for Innovation (CFI) grant to U. B. C. A.C. T. operates in the Parque Astronómico Atacama in northern Chile under the auspices of the Comisión Nacional de Investigación Científica y Tecnológica de Chile (CONICYT). Computations were performed on the GPC supercomputer at the SciNet HPC Consortium. SciNet is funded by the CFI under the auspices of Compute Canada, the Government of Ontario, the Ontario Research Fund Research Excellence, and the University of Toronto. The development of multichroic detectors and lenses was supported by NASA Grants No. NNX13AE56G and No. NNX14AB58G. N. S. acknowledges support from NSF Grant No. 1513618. A. K. has been supported by NSF Grant No. AST-1312380. R. D. and L. M. thank CONICYT for Grants No. ALMA-CONICYT 31140004, No. FONDECYT 
1141113, No. Anillo ACT-1417 and BASAL CATA. We also thank the Mishrahi Fund and the Wilkinson Fund for their generous support of the project.

\section{APPENDIX: THE TEMPERATURE CURL NULL TEST AND THE LOW- $\ell$ CUTOFF}

In our analysis, we impose a lower cutoff on the $\mathrm{CMB}$ scales $\ell_{\min }$, from which to measure our lensing. This cut was chosen to be above low multipoles where atmospheric

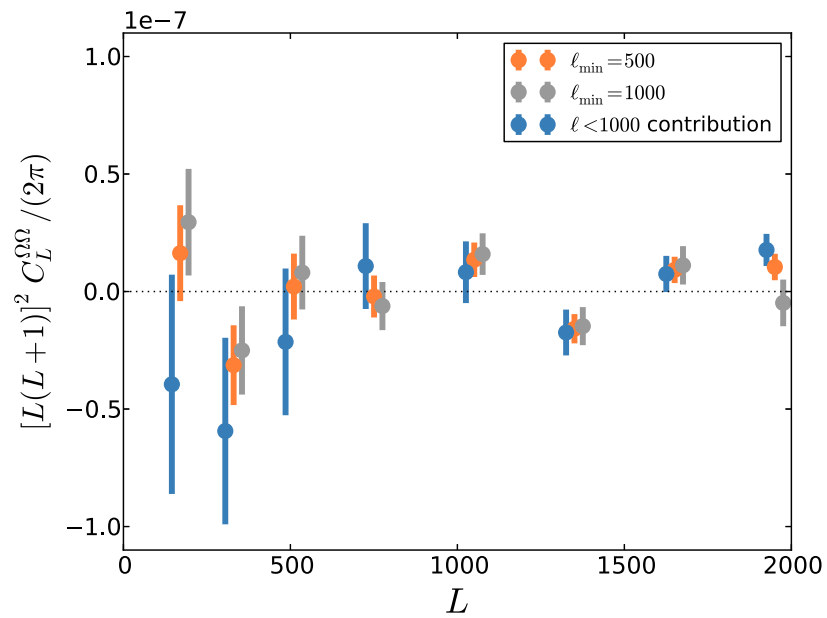

FIG. 10. Curl null test for the D56 TT, TT estimator, with different choices of the low-ell cutoff. It can be seen that the contribution from $\ell<1000$ is in $\sim 3 \sigma$ tension with null for the highest $L$ bandpower. This is likely due to the limitations of the realization-dependent bias subtraction, which only corrects for mismatched simulations to leading order, for the highest $L$ bandpower where the bias is very large-the simulations we use may not adequately capture the noise at $\ell<1000$ where atmospheric noise is very large. The problem is solved by, conservatively, using only CMB scales $\ell>1000$ in our analysis. noise is largest. Our initial choice was $\ell_{\min }=500$. However, with this initial choice, the curl null test for D56 for the $T T, T T$ estimator marginally fails at the $3 \sigma$ level (with a PTE of $\sim 0.2 \%$ ). Though with 60 null tests, one failure of this magnitude is not very unlikely (see discussion in the main text of the $T E, T E$ estimator); the D56 $T T, T T$ measurement is particularly important, as it dominates our result. A significant fraction of the tension seemed to arise from the highest $L$ bandpowers, approaching $L=2000$, where the Gaussian bias that we need to subtract off is largest.

As the prescription for calculating the realization dependent bias only self-corrects to first order in differences between simulations and data, we note that a mismatch in the simulated CMB power at the $10 \%$ level in any region of our map is sufficient to cause failures at the highest $L$. One possibility that gives such a mis-simulation is that our noise simulation procedure assumes that the atmospheric noise scales down with the local map weights as white noise, which is not quite true on large scales. We changed our cutoff to $\ell=1000$ in our analyses to address the fact that the bias subtraction might not be precise enough when using this range of scales in the estimator. This change results in the null test now passing at a slightly better than $2 \sigma$ level, because the highest $L$-bandpower is more consistent with null, though also to some extent because the error bars increased since data were removed. By varying the new cutoff above $\ell=1000$, we verified the stability of the result.

To check our understanding and to ensure that the scales we cut are at least partially responsible for the marginal null failure beyond merely inflating error bars, we plot the relevant null test points in Fig. 10 for both $\ell_{\min }=500$ and $\ell_{\text {min }}=1000$. We now seek to approximately isolate the new information arising from the low $\ell$ scales by assuming

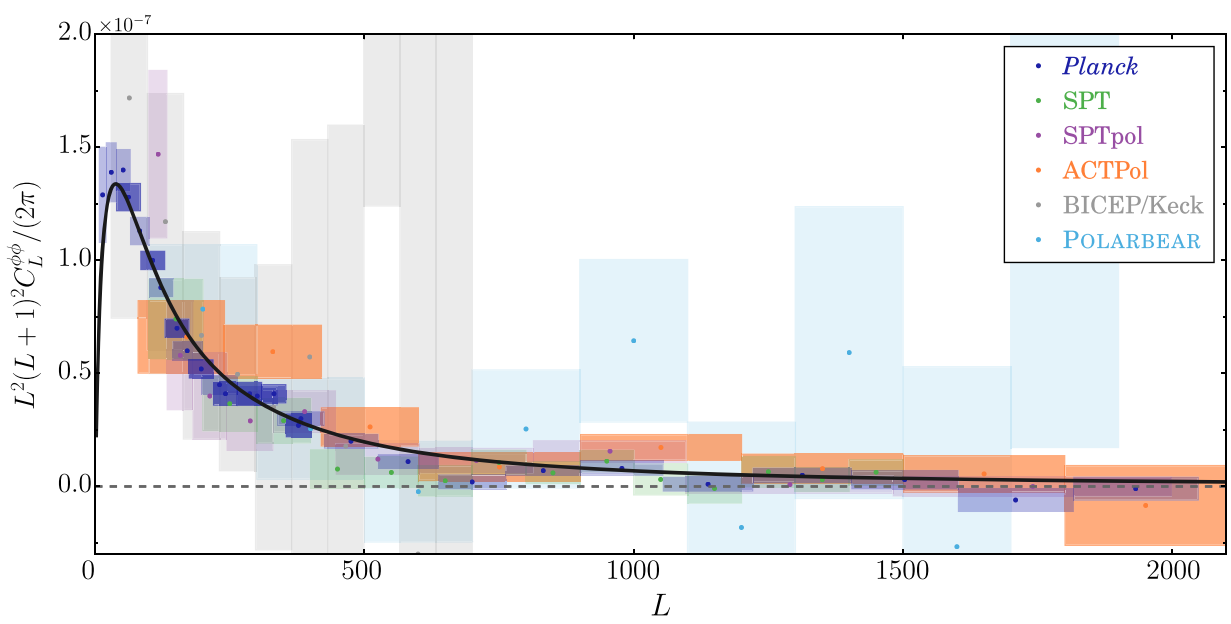

FIG. 11. Compilation of lensing power spectrum measurements from various experimental groups, including Planck [23], SPT [16], SPTpol [20], this work, BICEP/Keck [21], and Polarbear [17]. In the case of Planck, we show both the full range of reported bandpowers (light purple), as well as the restricted range $40<L<400$ used for cosmological analysis (dark purple). 
an independent measurement which is coadded with the $\ell_{\text {min }}=1000$ data to obtain the $\ell_{\text {min }}=500$ data. We invert the simple coadd procedure to obtain a new null test, which is also shown on the plot. We approximately identify this null with the contribution that we are cutting, i.e. the part that originates below $\ell_{\text {min }}=1000$ (noting that in noise domination with a realization dependent bias, the correlation of the four-point functions involving any $\ell<1000$ contribution with the $\ell_{\text {min }}=1000$ measurement is small).

It can be seen that the highest- $L$ bandpower deviates at the $3 \sigma$ level from null for the $\ell_{\text {min }}<1000$ contribution we isolate. This suggests that the large scales are to some extent responsible for the problem, and it is consistent with our picture of mis-simulation of atmospheric noise causing problems in the highest $L$ bandpower where the bias subtraction is largest. In a future work, we plan to prioritize our noise modeling (or alternatively, the development of a cross-spectrum based estimator) to mitigate this issue and extend the range of scales we can use in our analysis. In addition, with the significant increase in data expected from the full three-season data set, we will be able to investigate any hints of systematics in our data with more powerful null tests.
[1] A. Blanchard and J. Schneider, Astron. Astrophys. 184, 1 (1987).

[2] F. Bernardeau, Astron. Astrophys. 324, 15 (1997).

[3] M.Zaldarriaga and U. Seljak, Phys. Rev. D 59, 123507 (1999).

[4] A. Lewis and A. Challinor, Phys. Rep. 429, 1 (2006).

[5] A. Natarajan, A. R. Zentner, N. Battaglia, and H. Trac, Phys. Rev. D 90, 063516 (2014).

[6] T. Namikawa, Phys. Rev. D 93, 121301 (2016).

[7] J. Liu, J. C. Hill, B. D. Sherwin, A. Petri, V. Böhm, and Z. Haiman, Phys. Rev. D 94, 103501 (2016).

[8] V. Böhm, M. Schmittfull, and B. D. Sherwin, Phys. Rev. D 94, 043519 (2016).

[9] A. Kosowsky, New Astron. Rev. 47, 939 (2003).

[10] J. Ruhl, P. A. R. Ade, and J. E. Carlstrom et al., Proc. SPIE Int. Soc. Opt. Eng. 5498, 11 (2004).

[11] P. A. R. Ade, N. Aghanim et al. (Planck Collaboration), arXiv:astro-ph/0604069.

[12] K. M. Smith, O. Zahn, and O. Doré, Phys. Rev. D 76, 043510 (2007).

[13] C. M. Hirata, S. Ho, N. Padmanabhan, U. Seljak, and N. A. Bahcall, Phys. Rev. D 78, 043520 (2008).

[14] S. Das, B. D. Sherwin, P. Aguirre et al., Phys. Rev. Lett. 107, 021301 (2011).

[15] B. D. Sherwin, J. Dunkley, S. Das et al., Phys. Rev. Lett. 107, 021302 (2011).

[16] A. van Engelen, R. Keisler, O. Zahn et al., Astrophys. J. 756, 142 (2012).

[17] P. A. R. Ade, Y. Akiba, A. E. Anthony et al., Phys. Rev. Lett. 113, 021301 (2014).

[18] P. A. R. Ade, Y. Akiba, A. E. Anthony et al., Phys. Rev. Lett. 112, 131302 (2014).

[19] D. Hanson, S. Hoover, A. Crites et al., Phys. Rev. Lett. 111, 141301 (2013).

[20] K. T. Story, D. Hanson, P. A. R. Ade et al., Astrophys. J. 810, 50 (2015).

[21] Keck Array (BICEP2 Collaborations), Astrophys. J. 833, 228 (2016).

[22] P. A. R. Ade, N. Aghanim et al. (Planck Collaboration), Astron. Astrophys. 571, A17 (2014).

[23] P. A. R. Ade, N. Aghanim et al. (Planck Collaboration), Astron. Astrophys. 594, A15 (2016).
[24] H. Hildebrandt, M. Viola, C. Heymans et al., arXiv:1606.05338.

[25] F. De Bernardis, J. R. Stevens, M. Hasselfield et al., arXiv:1607.02120.

[26] A. van Engelen, B. D. Sherwin, N. Sehgal et al., Astrophys. J. 808, 7 (2015).

[27] R. Allison, S. N. Lindsay, B. D. Sherwin et al., Mon. Not. R. Astron. Soc. 451, 849 (2015).

[28] M. Madhavacheril, N. Sehgal et al., Phys. Rev. Lett. 114, 151302 (2015).

[29] R. J. Thornton, P. A. R. Ade, S. Aiola et al., Astrophys. J. Suppl. Ser. 227, 21 (2016).

[30] T. Louis, E. Grace, M. Hasselfield et al., arXiv:1610.02360.

[31] S. Naess, M. Hasselfield, J. McMahon et al., J. Cosmol. Astropart. Phys. 10 (2014) 007.

[32] M. Bucher and T. Louis, Mon. Not. R. Astron. Soc. 424, 1694 (2012).

[33] K. M. Smith, Phys. Rev. D 74, 083002 (2006).

[34] R. Pearson, B. Sherwin, and A. Lewis, Phys. Rev. D 90, 023539 (2014).

[35] E. Calabrese, R. A. Hlozek, N. Battaglia et al., Phys. Rev. D 87, 103012 (2013).

[36] T. Louis, S. Næss, S. Das, J. Dunkley, and B. Sherwin, Mon. Not. R. Astron. Soc. 435, 2040 (2013).

[37] T. Namikawa, D. Hanson, and R. Takahashi, Mon. Not. R. Astron. Soc. 431, 609 (2013).

[38] W. Hu and T. Okamoto, Astrophys. J. 574, 566 (2002).

[39] D. Hanson, A. Challinor, G. Efstathiou, and P. Bielewicz, Phys. Rev. D 83, 043005 (2011).

[40] M. M. Schmittfull, A. Challinor, D. Hanson, and A. Lewis, Phys. Rev. D 88, 063012 (2013).

[41] D. Green, J. Meyers, and A. van Engelen, arXiv:1609.08143.

[42] J. Peloton, M. Schmittfull, A. Lewis, J. Carron, and O. Zahn, Phys. Rev. D 95, 043508 (2017).

[43] G. Pratten and A. Lewis, J. Cosmol. Astropart. Phys. 8 (2016) 047.

[44] G. Marozzi, G. Fanizza, E. Di Dio, and R. Durrer, J. Cosmol. Astropart. Phys. 9 (2016) 028.

[45] S. J. Osborne, D. Hanson, and O. Doré, J. Cosmol. Astropart. Phys. 3 (2014) 024. 
[46] A. van Engelen, S. Bhattacharya, N. Sehgal, G. P. Holder, O. Zahn, and D. Nagai, Astrophys. J. 786, 13 (2014).

[47] N. Sehgal, P. Bode, S. Das, C. Hernandez-Monteagudo, K. Huffenberger, Y.-T. Lin, J. P. Ostriker, and H. Trac, Astrophys. J. 709, 920 (2010).

[48] J. Hartlap, P. Simon, and P. Schneider, Astron. Astrophys. 464, 399 (2007).

[49] R. E. Smith, J. A. Peacock, A. Jenkins, S. D. M. White, C. S. Frenk, F. R. Pearce, P. A. Thomas, G. Efstathiou, and H. M. P. Couchman, Mon. Not. R. Astron. Soc. 341, 1311 (2003).

[50] R. Takahashi, M. Sato, T. Nishimichi, A. Taruya, and M. Oguri, Astrophys. J. 761, 152 (2012).

[51] A. Lewis, Phys. Rev. D 87, 103529 (2013).

[52] A. Lewis and S. Bridle, Phys. Rev. D 66, 103511 (2002).

[53] R. Adam, N. Aghanim et al. (Planck Collaboration), Astron. Astrophys. 596, A108 (2016).

[54] M. Pettini and R. Cooke, Mon. Not. R. Astron. Soc. 425, 2477 (2012).

[55] P. A. R. Ade, N. Aghanim et al. (Planck Collaboration), Astron. Astrophys. 594, A13 (2016).
[56] F. Beutler, C. Blake, M. Colless, D. H. Jones, L. StaveleySmith, L. Campbell, Q. Parker, W. Saunders, and F. Watson, Mon. Not. R. Astron. Soc. 416, 3017 (2011).

[57] A. J. Ross, L. Samushia, C. Howlett, W. J. Percival, A. Burden, and M. Manera, Mon. Not. R. Astron. Soc. 449, 835 (2015).

[58] H. Gil-Marín, W. J. Percival, A. J. Cuesta et al., Mon. Not. R. Astron. Soc. 460, 4210 (2016).

[59] S. Joudaki, C. Blake, C. Heymans et al., Mon. Not. R. Astron. Soc. 465, 2033 (2017).

[60] H. Hildebrandt, M. Viola, C. Heymans et al., arXiv: 1606.05338.

[61] T. Abbott, F. B. Abdalla et al. (Dark Energy Survey Collaboration), Phys. Rev. D 94, 022001 (2016).

[62] G. E. Addison, Y. Huang, D. J. Watts, C. L. Bennett, M. Halpern, G. Hinshaw, and J. L. Weiland, Astrophys. J. 818, 132 (2016).

[63] N. Aghanim, Y. Akrami et al. (Planck Collaboration), arXiv:1608.02487.

[64] K. A. Olive et al. (Particle Data Group Collaboration), Chin. Phys. C 38, 090001 (2014).

[65] E. Calabrese, A. Slosar, A. Melchiorri, G. F. Smoot, and O. Zahn, Phys. Rev. D 77, 123531 (2008). 